Research Article

\title{
Applications of Homogenous Balance Principles Combined with Fractional Calculus Approach and Separate Variable Method on Investigating Exact Solutions to Multidimensional Fractional Nonlinear PDEs
}

\author{
Ruichao Ren ${ }^{(D)}{ }^{1}$ Shunli Zhang, ${ }^{2}$ and Weiguo Rui ${ }^{3}{ }^{3}$ \\ ${ }^{1}$ School of Mathematics, Northwest University, Xi'an 710127, China \\ ${ }^{2}$ Center for Nonlinear Studies, Northwest University, Xi'an 710069, China \\ ${ }^{3}$ School of Mathematical Sciences, Chongqing Normal University, Chongqing 401331, China
}

Correspondence should be addressed to Ruichao Ren; rrc8512@163.com

Received 27 November 2019; Revised 15 February 2020; Accepted 3 March 2020; Published 6 May 2020

Academic Editor: Krzysztof Kecik

Copyright (C) 2020 Ruichao Ren et al. This is an open access article distributed under the Creative Commons Attribution License, which permits unrestricted use, distribution, and reproduction in any medium, provided the original work is properly cited.

We investigate the exact solutions of multidimensional time-fractional nonlinear PDEs (fnPDEs) in this paper. In terms of the fractional calculus properties and the separate variable method, we present a new homogenous balance principle (HBP) on the basis of the $(1+1)$-dimensional time fnPDEs. Taking advantage of the new types of HBP together with fractional calculus formulas that subtly avoid the chain rule, the fnPDEs can be reduced to spatial PDEs, and then we solve these PDEs by the fractional calculus method and the separate variable approach. In this way, some new type exact solutions of the certain time-fractional $(2+1)$ dimensional KP equation, (3 + 1)-dimensional Zakharov-Kuznetsov (ZK) equation, and Jimbo-Miwa (JM) equation are explicitly obtained under both Riemann-Liouville derivatives and Caputo derivatives. The dynamical analysis of solutions is shown by numerical simulations of taking property parameters as well.

\section{Introduction}

The fractional PDEs (fPDEs) have been more and more widely followed with interest up to now since they can be used to accurately describe many nonlinear stochastic phenomena which depend on both time instant and the previous time history in the real-time problem [1-4]. Models set up from the fPDEs play very important roles in a range of scientific fields, such as viscoelastic flow $[5,6]$, signal processing $[7,8]$, control systems $[9,10]$, material diffusion including normal diffusion and anomalous diffusion(superdiffusion, subdiffusion, fast diffusion, and slow diffusion) [11-15], biological mathematics [16, 17], and magnetohydrodynamics (MHD) $[18,19]$. Similarly, as some time-fractional PDEs, the space fractional models are also very frequently used in some elastic materials (see [20-24]) in the field of mechanical engineering. Therefore, investigating their solutions also draws much attention from the mathematical and physical points of view, and it can help to concisely characterize and well understand the qualitative features of the concerned phenomena and nonlinear processes in various areas of natural science and engineering, which involved the ubiquitous time memory effects $[20,21]$ (including some short memories) and space viscoelastic effects (see [22-26]), in particular many complex random walks and material motions in the microscopic space, such as dynamical behaviors of fractional diffusion, particles spread in heat bath, and soft matter interaction with viscoelasticity.

In the past few years, there were excessive studies on the $(1+1)$-dimensional fractional nonlinear PDEs(fnPDEs), and a lot of excellent tools were used for solving them, which include Adomian decomposition method [27, 28], homotopy analysis method (HAM) $[29,30]$, fractional variational 
method [31, 32], Lie symmetry method [33-40], invariant subspace method [40-44], and homogenous balance principle (HBP) [45, 46]. Recently, a few of the above methods were also applied to solve several $(2+1)$-dimensional fnPDEs [47-50]. Although approximate analytic solutions or exact solutions of some $(1+1)$-dimensional fnPDEs and $(2+1)$-dimensional fnPDEs can be successfully obtained by using the above methods, this is far from enough, and these methods still have many limitations in solving more complex multidimensional fnPDEs. In this paper, we suggest a new technique to solve the following type of fnPDEs with certain time-fractional derivatives:

$$
\partial_{t}^{\alpha} u=F\left(x, t, u, \partial_{x} u, \ldots, \partial_{x}^{m} u\right)
$$

where $\quad x=\left(x_{1}, \ldots, x_{N}\right), \partial_{x}^{k} u=\left(\partial^{i_{1}+\cdots+i_{N}} u / \partial x_{1}^{i_{1}} \ldots \partial x_{N}^{i_{N}}\right)$, $\left(1 \leq k \leq m, 1 \leq i_{1}+i_{N} \leq k\right)$, and the index $0<\alpha \leq 1$. The $\alpha$-th order time derivative $(\alpha \in(0,1])$ is well defined as an abnormal derivative in the real applications for explaining short memories of evolutional physical systems; there are several kinds of definitions of fractional derivatives, and the two frequently used, classical, and very widely influential definitions are still Riemann-Liouville definition and Caputo definition (see Definitions 1 and 2). Indeed, with the help of Riemann-Liouville derivative and Caputo derivative, the results derived from the time-fractional PDE models are more precise and more general in nature than those of the integer-order ones, while the smaller the $\alpha$ is, the faster effective time memory enjoys [20, 21]. When $\alpha=1$, expressions (3) and (4) are exactly in accordance with the classical derivatives $(\partial u / \partial t)$; however, compared with the Riemann-Liouville type of derivative, the Caputo type of derivative possesses weaker singularity for handling some fractional initial problems.

Moreover, it is necessary to point out that the two travelling wave transformations $u=U(\xi)\left(\xi=\sum_{i=1}^{N} k_{i} x_{i}-\right.$ $\left.\omega\left(t^{\alpha} / \Gamma(1+\alpha)\right)\right)$ and $u=U(\xi)\left(\xi=\sum_{i=1}^{N} k_{i} x_{i}-\omega t\right)$, which were often used to reduce the integer-order PDE to ODE, are actually not valid for the fractional-order one since it had been successfully verified that the compound fractional derivatives disagree with the following chain rule (refer to $[45,46,51,52])$ :

$$
D_{x}^{\alpha} f(g(x))=f^{\prime}(g(x)) D_{x}^{\alpha} g(x)=D_{g(x)}^{\alpha} f(g(x))\left(g^{\prime}(x)\right)^{\alpha},
$$

thus, we hardly take the fractional derivative of compound function straightly in accordance with the classical chain rule, and the normal chain rule (see [51]) of fractional derivatives was ineffectively applied to solve equation (1) since it contains infinite series. That is to say, the exact solutions of the compound function type of equation (1) were impossible to be obtained by the invalid chain rule (2), and we hardly find the travelling wave solutions (even the exact soliton solutions) of fnPDEs via the two transformations mentioned above or some other complex transformations such as Darboux transformation [53], bilinear method [54], and F-expansion method [55].

To the best of our knowledge, avoiding the invalid fractional chain rule (2), there is no more direct and effective method to obtain the exact solutions for equation (1). The exact solutions of higher-dimensional nonlinear PDEs with time-fractional derivatives were not well obtained. The main difficulty is how to construct solutions to reduce the multidimensional fnPDEs to the classical spatial PDEs. Inspired by the previous homogenous balance principle (HBP) [45-47] including fractional calculus method [40-44, 56] and separate variable approach, we improve the way [45-47] and introduce a new type of HBPs for (1) so that the solutions can be assumed as a general separated variable form and (1) can be reduced to spatial PDEs, and then new type exact solutions of some multidimensional fnPDEs will be successfully obtained by solving these reduced PDEs in the variable separate way under both Riemann-Liouville derivatives and Caputo derivatives.

The main contents of this paper are organized as follows. The definitions and properties of the fractional calculus and fractional Laplace transformation are briefly described in Section 2. In Section 3, based on the fractional derivative formulas and the method of separate variable, the new homogenous balance principle (HBP) is suggested for (1), and in this way, the certain time-fractional $(2+1)$-dimensional KP equation, $(3+1)$-dimension Zakharov-Kuznetsov (ZK) equation, and $(3+1)$-dimension Jimbo-Miwa (JM) equation are reduced to the spatial PDEs and explicitly solved in general separated variable forms; we can see that some of these solutions possess new types including some arbitrary functions which were never attained before by other way, which means more general solutions are obtained. Furthermore, there are real differences between the Riemann-Liouville case and the Caputo case: the singularity occurs in solutions under Riemann-Liouville derivatives but no singularity appears in solutions under Caputo derivatives. The dynamical profiles of these solutions are displayed as can be seen from Figures 1-13 with property parameters, and we also analyze the long time behaviors for some of them as well. The last section is the conclusions of our works.

\section{Preliminaries}

In this section, we recall some useful definitions, properties, and theorem.

\subsection{Definitions and Properties of Two Type Fractional Derivatives}

Definition 1. The Riemann-Liouville fractional derivative of order $\alpha>0$ is defined by the following expression: 


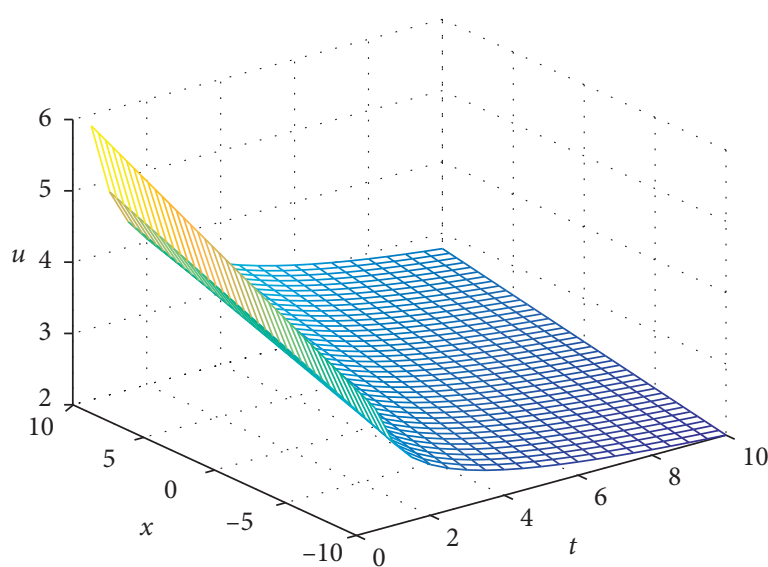

(a)

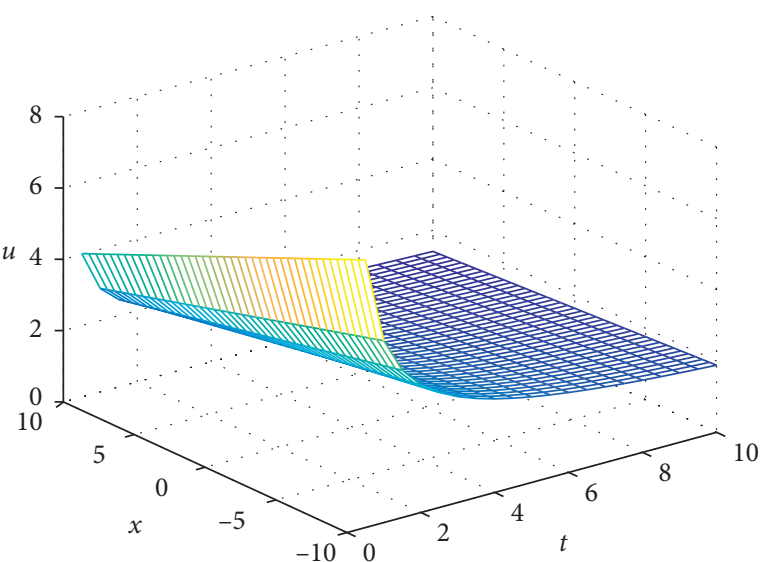

(b)

FIgURE 1: Dynamical profiles of solution (28) on the $(x, t, u)$-plane when (a) $\alpha=(1 / 3)$ and (b) $\alpha=(2 / 3)$.

$$
\mathrm{RL}_{t}^{\alpha} u(x, t)= \begin{cases}\frac{1}{\Gamma(n-\alpha)} \frac{\partial^{n}}{\partial t^{n}} \int_{0}^{t}(t-\tau)^{n-\alpha-1} u(x, \tau) \mathrm{d} \tau, & (n=[\alpha]+1), \\ \frac{\partial^{n} u(x, t)}{\partial t^{n}}, & (n=\alpha) .\end{cases}
$$

Definition 2. The Caputo fractional derivative of order $\alpha>0$

is defined by the following expression:

$$
{ }^{C} \partial_{t}^{\alpha} u(x, t)= \begin{cases}\frac{1}{\Gamma(n-\alpha)} \int_{0}^{t}(t-\tau)^{n-\alpha-1} \frac{\partial^{n} u(x, \tau)}{\partial \tau^{n}} \mathrm{~d} \tau, & (n=[\alpha]+1), \\ \frac{\partial^{n} u(x, t)}{\partial t^{n}}, & (n=\alpha) .\end{cases}
$$

Proposition 1. Some properties of the fractional derivative:

(i) ${ }^{R} D_{t}^{\alpha} c=c\left(t^{-\alpha} / \Gamma(1-\alpha)\right)$ (c is a constant)

(ii) ${ }^{C} D_{t}^{\alpha} c=0$ (c is a constant)

(iii) $D_{t}^{\alpha}\left(\sum_{k=1}^{n} c_{k} f_{k}(t)\right)=\sum_{k=1}^{n} c_{k} D_{t}^{\alpha} f_{k}(t) \quad\left(c_{k} \quad\right.$ are constants)

(iv) $D_{t}^{\alpha} t^{\mu}=(\Gamma(1+\mu) / \Gamma(1+\mu-\alpha)) t^{\mu-\alpha},(\mu \neq \alpha-1)$ holds for $\mu>-1$ under the Riemann-Liouville case and $\mu>0$ under the Caputo case (5).
Proposition 2. The rules of the partial fractional derivative of a separate variable form:

(i) For $\varphi(t) \neq$ Const, $D_{t}^{\alpha} f(x) \varphi(t)=f(x) D_{t}^{\alpha} \varphi(t)$.

(ii) For $\varphi(t)=1$,

$$
D_{t}^{\alpha} f(x)= \begin{cases}f(x) \frac{t^{-\alpha}}{\Gamma(1-\alpha)}, & \text { (under Riemann-Liouville derivative) } \\ 0, & \text { (under Caputo derivative). }\end{cases}
$$

\subsection{Definitions of Mittag-Leffler Function and Some Properties}

Definition 3. The Mittag-Leffler function is defined by the infinity series expression:

$$
E_{\alpha, \beta}(z)=\sum_{k=0}^{\infty}\left(\frac{z^{k}}{\Gamma(\alpha k+\beta)}\right),
$$

with $\alpha>0$ and $\beta>0$. 
Proposition 3. The properties of the Mittag-Leffler function:

(i) $E_{1,1}(z)=e^{z}$

(ii) $D_{t}^{\gamma}\left(t^{(\alpha k+\beta-1)} E_{\alpha, \beta}^{(k)}\left(\lambda t^{\alpha}\right)\right)=t^{(\alpha k+\beta-\gamma-1)} E_{\alpha, \beta-\gamma}^{(k)}\left(\lambda t^{\alpha}\right)$,

(iii) ${ }^{C} D_{t}^{\alpha} E_{\alpha, 1}\left(\lambda t^{\alpha}\right)=\lambda E_{\alpha, 1}\left(\lambda t^{\alpha}\right)$,

(iv) ${ }^{R} D_{t}^{\alpha} E_{\alpha, 1}\left(\lambda t^{\alpha}\right)=\left(t^{-\alpha} / \Gamma(1-\alpha)\right)+\lambda E_{\alpha, 1}\left(\lambda t^{\alpha}\right)$,

(v) The Mittag-Leffler function $E_{\alpha, 1}\left(\lambda t^{\alpha}\right)$ is increasing as $\lambda>0$ while decreasing as $\lambda<0$ when $\alpha>0$ (Definition 3).

The brief proof of (iii) and (iv) is given as follows. For the Caputo derivatives, we have

$$
\begin{aligned}
{ }^{C} D_{t}^{\alpha} E_{\alpha, 1}\left(\lambda t^{\alpha}\right) & ={ }^{C} D_{t}^{\alpha} \sum_{k=0}^{\infty} \frac{\left(\lambda t^{\alpha}\right)^{k}}{\Gamma(\alpha k+1)}=\sum_{k=0}^{\infty} \frac{\lambda^{k}\left({ }^{C} D_{t}^{\alpha} t^{k \alpha}\right)}{\Gamma(\alpha k+1)} \\
& =\lambda \sum_{k=1}^{\infty} \frac{\lambda^{k-1}(\Gamma(\alpha k+1) / \Gamma(\alpha k+1-\alpha)) t^{(k-1) \alpha}}{\Gamma(\alpha k+1)} \\
& =\lambda \sum_{n=0}^{\infty} \frac{\left(\lambda t^{\alpha}\right)^{n}}{\Gamma(\alpha n+1)} \\
& =\lambda E_{\alpha, 1}\left(\lambda t^{\alpha}\right) .
\end{aligned}
$$

For the Riemann-Liouville derivatives, we obtain

$$
\begin{aligned}
{ }^{R} D_{t}^{\alpha} E_{\alpha, 1}\left(\lambda t^{\alpha}\right) & ={ }^{R} D_{t}^{\alpha} \sum_{k=0}^{\infty} \frac{\left(\lambda t^{\alpha}\right)^{k}}{\Gamma(\alpha k+1)}=\sum_{k=0}^{\infty} \frac{\lambda^{k}\left({ }^{R} D_{t}^{\alpha} t^{k \alpha}\right)}{\Gamma(\alpha k+1)} \\
& =\frac{t^{-\alpha}}{\Gamma(1-\alpha)}+\lambda \sum_{k=1}^{\infty} \frac{\lambda^{k-1}(\Gamma(\alpha k+1) / \Gamma(\alpha k+1-\alpha)) t^{(k-1) \alpha}}{\Gamma(\alpha k+1)} \\
& =\frac{t^{-\alpha}}{\Gamma(1-\alpha)}+\lambda \sum_{n=0}^{\infty} \frac{\left(\lambda t^{\alpha}\right)^{n}}{\Gamma(\alpha n+1)}=\frac{t^{-\alpha}}{\Gamma(1-\alpha)}+\lambda E_{\alpha, 1}\left(\lambda t^{\alpha}\right) .
\end{aligned}
$$

These achieve (iii) and (iv) in Proposition 3.

\section{HBP for Some Multidimensional Time- Fractional PDEs}

In the beginning of this section, we improve the previous way to construct exact solutions to some multidimensional time-fractional nonlinear PDEs (1) according to ideas of homogenous balance and the fractional properties (5).

The main procedures are stated as follows:

Step 1. As can be seen from the properties of power function and Mittag-Leffler function in Section 2 and motivated by the HBP of $(1+1)$-dimensional timefractional nonlinear PDE, for equation (1), we suppose the exact solutions as the following two types:

(1) For the Riemann-Liouville case:

$$
u=a_{0} t^{\mu_{1}}+a_{1} v\left(x_{1}, \ldots, x_{N}\right) t^{\mu_{2}}, \quad \mu_{1}, \mu_{2} \neq \alpha-1 .
$$

(2) For the Caputo case:

$$
u=a_{0}+a_{1} v\left(x_{1}, \ldots, x_{N}\right) E_{\alpha, 1}\left(\lambda t^{\alpha}\right)
$$

Here $\mu_{1}, \mu_{2}$, and $\lambda$ are undetermined parameters and $v\left(x_{1}, \ldots, x_{N}\right)$ is a undetermined function.

Step 2. Substituting (9) or (10) into equation (1) by comparing the powers of $t$ or the coefficients of $E_{\alpha, 1}\left(\lambda t^{\alpha}\right)$, we have parameters $\mu_{1}, \mu_{2}$, or $\lambda$ and the reduced spatial PDE system for $v\left(x_{1}, \ldots, x_{N}\right)$ :

$$
v\left(x_{1}, \ldots, x_{N}\right)=\Phi\left(x, t, v, \partial_{x} v, \ldots, \partial_{x}^{m} v\right),
$$

where

$x=\left(x_{1}, \ldots, x_{N}\right), \partial_{x}^{k} v=\frac{\partial^{i_{1}+\cdots+i_{N}} v}{\partial x_{1}^{i_{1}} \ldots \partial x_{N}^{i_{N}}}, \quad 1 \leq k \leq m, 1 \leq i_{1}+i_{N} \leq k$.

Step 3. Solving the reduced PDE system (10) by using complex transformation $\xi=c_{0}+\sum_{i=1}^{N} c_{i} x_{i}$ or a separate variable approach (in product or summary form) leads to the exact solutions.

Remark 1. When $\mu_{1}, \mu_{2}=\alpha-1$, singular solutions will appear under the Riemann-Liouville derivative and while solutions appear, no singularity will be obtained in the Caputo sense. $t$ should involve minus power, thereby the assumption (9) fits for the Riemann-Liouville derivative, and since the fractional derivative of Mittag-Leffler function includes an additional term $\left(t^{-\alpha} / \Gamma(1-\alpha)\right)$ under the Riemann-Liouville derivative, this will complicate the calculation of solutions, and the assumption (10) fits for the Caputo derivative.

We will study time-fractional $(2+1)$-dimensional KP equation, $(3+1)$-dimensional ZK equation, and JM equation in the following paragraphs.

3.1. HBP for the $(2+1)$-Dimensional Time-Fractional KP Equation. We first begin with the $(2+1)$-dimensional fractional KP equation:

$$
\left(\partial_{t}^{\alpha} u-6 u u_{x}+u_{x x x}\right)_{x}+3 u_{y y}=0, \quad 0<\alpha \leq 1,
$$

which developed from the classical KdV equation, describes long dispersive wave propagating in two dimension in shallow water.

3.1.1. Exact Solutions under Riemann-Liouville Derivatives. According to (9), we suppose the exact solutions as

$$
u=a_{0} t^{\mu_{1}}+a_{1} v(x, y) t^{\mu_{2}},
$$

where $a_{0}, a_{1}, \mu_{1}$, and $\mu_{2}$ are the undetermined constants.

Balancing the coefficients of $t$-power yields the following two subcases:

Subcase I

$$
\mu_{2}-\alpha=\mu_{1}+\mu_{2}=2 \mu_{2}
$$


Thus, $\mu_{1}=\mu_{2}=-\alpha$.

Substituting (15) into (14) by comparing the coefficients of $t^{-\alpha}$ and $t^{-2 \alpha}$ yields

$$
\begin{aligned}
& \Omega_{0} v_{x}= 6\left(a_{0}+a_{1} v\right) v_{x x}+6 a_{1} v_{x}^{2},\left(\Omega_{0}=\frac{\Gamma(1-\alpha)}{\Gamma(1-2 \alpha)}\right), \\
& v_{x x x x}+3 v_{y y}=0 .
\end{aligned}
$$

Then, integrating (16a) corresponding to variable $x$ once by selecting an integrate constant 0 leads to

$$
\frac{\mathrm{d} v}{\mathrm{~d} x}=\frac{\Omega_{0} v}{6\left(a_{0}+a_{1} v\right)}
$$

This gives rise to the implicit relation:

$$
6\left(a_{0} \ln v+a_{1} v\right)=\Omega_{0}(x+\phi(y)),
$$

where $\phi(y)$ is an undetermined function.

Calculating the derivatives of implicit function (18) reads that

$$
\begin{aligned}
v_{x x x x} & =\frac{a_{0} \Omega_{0}^{4}\left(6 a_{1}^{2} v^{2}-8 a_{0} a_{1} v+a_{0}^{2}\right) v}{1296\left(a_{1}^{7} v^{7}+7 a_{0} a_{1}^{6} v^{6}+21 a_{0}^{2} a_{1}^{5} v^{5}+35 a_{0}^{3} a_{1}^{4} v^{4}+35 a_{0}^{4} a_{1}^{3} v^{3}+21 a_{0}^{5} a_{1}^{2} v^{2}+7 a_{0}^{6} a_{1} v+a_{0}^{7}\right)}, \\
v_{y y} & =\frac{\Omega_{0}\left(\Omega_{0} a_{0} \phi^{\prime}(y)^{2}+6\left(a_{0}+a_{1} v\right)^{2} \phi^{\prime \prime}(y) v^{2}\right) v}{36\left(a_{1}^{3} v^{3}+3 a_{0} a_{1}^{2} v^{2}+3 a_{0}^{2} a_{1} v+a_{0}^{3}\right)} .
\end{aligned}
$$

Plugging (19a) and (19b) into (15), we have

$$
\begin{aligned}
a_{0} & =0, \\
\phi^{\prime \prime}(y) & =0,
\end{aligned}
$$

which leads to the solution of (15)

$$
v(x, y)=\frac{\Omega_{0}}{6 a_{1}}\left(x+b_{1} y+b_{2}\right)
$$

with arbitrary constants $a_{1}, b_{1}$, and $b_{2}$ and the exact solution of (11)

$u(t, x, y)=\left[a_{0}+\frac{\Gamma(1-\alpha)}{6 \Gamma(1-2 \alpha)}\left(x+b_{1} y+b_{2}\right)\right] t^{-\alpha}, \quad\left(\alpha \neq \frac{1}{2}\right)$,

where $c_{1}$ and $c_{2}$ are the arbitrary constants.

Subcase II. $\mu_{2}-\alpha=2 \mu_{2}$ and $\mu_{1}+\mu_{2}=\mu_{2}$.

Thus, $\mu_{1}=0$ and $\mu_{2}=-\alpha$.

Similarly as above, substituting (13) into (11) by comparing the coefficients of $t^{-\alpha}$ and $t^{-2 \alpha}$ yields

$$
\begin{aligned}
& \Omega_{0} v_{x}=6 a_{1} v v_{x x}+6 a_{1} v_{x}^{2},\left(\Omega_{0}=\frac{\Gamma(1-\alpha)}{\Gamma(1-2 \alpha)}\right), \\
& v_{x x x x}+6 a_{0} v_{x x}+3 v_{y y}=0 .
\end{aligned}
$$

Then, integrating (23a) corresponding to variable $x$ by choosing an integrate constant 0 once leads to

$$
\Omega_{0} v=6 a_{1} v v_{x}
$$

Since $v \neq 0$ (only nontrivial solutions are considered), we have

$$
\frac{\mathrm{d} v}{\mathrm{~d} x}=\frac{\Omega_{0}}{6 a_{1}}
$$

which give rises to

$$
v=\frac{\Omega_{0}}{6 a_{1}} x+\phi(y)
$$

Plugging (26) into (23b) yields

$$
\phi(y)=c_{1} y+c_{2}
$$

By rewriting $b_{1}=a_{1} c_{1}$ and $b_{2}=a_{2} c_{2}$, we have the exact solution

$$
u(t, x, y)=a_{0}+\left(\frac{\Gamma(1-\alpha)}{6 \Gamma(1-2 \alpha)} x+b_{1} y+b_{2}\right) t^{-\alpha}, \quad\left(\alpha \neq \frac{1}{2}\right)
$$

where $a_{0}, b_{1}$, and $b_{2}$ are the arbitrary constants.

Remark 2. We have the same type solutions under the complex transformation $\xi=c_{1} x+c_{2} y+c_{0}$, so this case is omitted here.

3.1.2. Exact Solutions under Caputo Derivatives. According to (10), we assume the exact solutions formed as

$$
u=a_{0}+a_{1} v(x, y) E_{\alpha, 1}\left(\lambda t^{\alpha}\right)
$$

Substituting (29) into (11) by comparing the coefficients of $E_{\alpha, 1}\left(\lambda t^{\alpha}\right)$ leads to 


$$
\begin{aligned}
\lambda a_{1} v_{x}+a_{1} v_{x x x x}+a_{0} v_{x x}+3 a_{1} v_{y y} & =0, \\
v v_{x x} & =v_{x}^{2} .
\end{aligned}
$$

We consider the following two cases:

Case I. By using the total differential rule, from (30b), we have

$$
\frac{v v_{x x}-v_{x}^{2}}{v^{2}}=\left(\frac{v_{x}}{v}\right)_{x}=0 .
$$

Integrating (31) leads to

$$
v_{x}=C(y) v
$$

Thus,

$$
v=\phi(y) e^{C(y) x}
$$

where $\phi(y)$ and $C(y)$ are the undetermined functions. Plugging (33) into (30a) yields

$$
\phi^{\prime \prime}(y)+\frac{c \lambda+c^{4}+a_{0} c^{2}}{3} \phi(y)=0 .
$$

Then, we have following three type solutions:

(i) $\delta=c \lambda+c^{4}+a_{0} c^{2}<0$

We obtain $\phi(y)=c_{1} e^{-\sqrt{-(\delta / 3)} y}+c_{2} e^{\sqrt{-(\delta / 3)} y}$ and the exact solution

$u(t, x, y)=a_{0}+\left(c_{1} e^{c x-\sqrt{-(\delta / 3)} y}+c_{2} e^{c x+\sqrt{-(\delta / 3)} y}\right) E_{\alpha, 1}\left(\lambda t^{\alpha}\right)$

where $a_{0}, c, c_{1}$, and $c_{2}$ are the arbitrary constants.

(ii) $\delta=c \lambda+c^{4}+a_{0} c^{2}>0$.

We obtain the exact solution

$$
\begin{aligned}
u(t, x, y)= & a_{0}+\left(c _ { 1 } \operatorname { c o s } \left(\sqrt{\left.\frac{\delta}{3} y\right)}\right.\right. \\
& \left.+c_{2} \sin \left(\sqrt{\frac{\delta}{3}} y\right)\right) e^{c x} E_{\alpha, 1}\left(\lambda t^{\alpha}\right),
\end{aligned}
$$

where $a_{0}, c, c_{1}$, and $c_{2}$ are the arbitrary constants.

(iii) $\delta=c \lambda+c^{4}+a_{0} c^{2}=0$.

We obtain $\phi(y)=c_{1} y+c_{2}$ and the exact solution

$$
u(t, x, y)=a_{0}+\left(c_{1} y+c_{2}\right) e^{c x} E_{\alpha, 1}\left(\lambda t^{\alpha}\right)
$$

where $a_{0}, c, c_{1}$, and $c_{2}$ are the arbitrary constants.

Case II. Using the complex differential transformation $\xi=c_{1} x+c_{2} y+c_{0}$ on (30a) and (30b), we have

$$
\begin{aligned}
c_{1}^{4} v^{(4)}(\xi)+\left(a_{0} c_{1}^{2}+3 c_{2}^{2}\right) v^{\prime \prime}(\xi)+\lambda c_{1} v^{\prime}(\xi) & =0, \\
v(\xi) v^{\prime \prime}(\xi) & =v^{\prime 2}(\xi) .
\end{aligned}
$$

Solving (38b) similarly as (25) yields

$$
v(\xi)=r e^{c \xi}
$$

Taking (39) into (38a), we obtain $c_{1}^{4} c^{3}+\left(a_{0} c_{1}^{2}+3 c_{2}^{2}\right) c+$ $\lambda c_{1}=c_{0}$ and the exact solution by rewriting $a=a_{1} r e^{c c_{0}}, b_{1}=c c_{1}, b_{2}=c c_{2}$

$$
u(t, x, y)=a_{0}+a e^{\left(b_{1} x+b_{2} y\right)} E_{\alpha, 1}\left(\lambda t^{\alpha}\right),
$$

where $a_{0}, a, b_{1}$, and $b_{2}$ are the arbitrary constants.

Remark 3. (33) contains product forms of separate variable $v=f(x) g(y)$, and for omitting the trivial solution, we do not consider the summary form of a separate variable $v=f(x) g(y)$.

Remark 4 . The singularity only appears when $\alpha=(1 / 2)$ in solutions (22) and (28) in the Riemann-Liouville case.

\subsubsection{Dynamical Analysis of Exact Solution for Fractional KP} Model. Under the Riemann-Liouville case:

As can be seen from Figure 1, taking parameters $\alpha=$ $(1 / 3)$ and $\alpha=(2), a_{0}=1, b_{1}=2, b_{2}=1, y=1$, and interval $x \in[-10,10], t \in[0,10]$, it is shown that solution (28) increases with increase in the spatial variable and decreases with increase in time. When $x$ and $y$ are fixed, the solution tends to 0 at an $\alpha$ decay rate as $t \longrightarrow \infty$, the larger the $\alpha$ is, the faster the $u$ decay is.

Under the Caputo case:

(i) As can be seen from Figure 2, choosing $c_{1}=1, c_{2}=$ $-2, c=1, \lambda=-2, \alpha=(1 / 2), \delta=-1, y=2, x \in$ $[-10,10], t \in[0,10]$, and $x=2, y \in[-10,10], t \in$ $[0,10]$, it is shown that solution (35) decreases with increase in time-space since slope $\left(u_{x}<0\right)$ or $\left(u_{y}<0\right)$; however, it is unbounded as $x$ or $y$ tends to $\infty$.

(ii) As we see from Figure 3, selecting $c_{1}=1, c_{2}=-2, c=2, \lambda=12, \alpha=(1 / 2), \delta=-1, y=$ $2, x \in[-10,10], t \in \quad[0,10], \quad$ and $\quad x=2, y \in$ $[-30,10], t \in[0,10]$, solution (36) decreases as time increases, periodic bounded as $y, t$ tends to $\infty$ if $x$ is fixed while unbounded as $x$, and $t$ tends to $\infty$ if $y$ is fixed.

(iii) As we see from Figure 4 , choosing $c_{1}=1, c_{2}=-2, c=$ $1, \lambda=-1, \alpha=(1 / 2), \delta=0, y=3, x \in[-10,10], t \in[0,10]$, and $x=2, y \in[-10,10], t \in[0,10]$, solution (37) decreases as time increases and unbounded as timespace tend to $\infty$. 


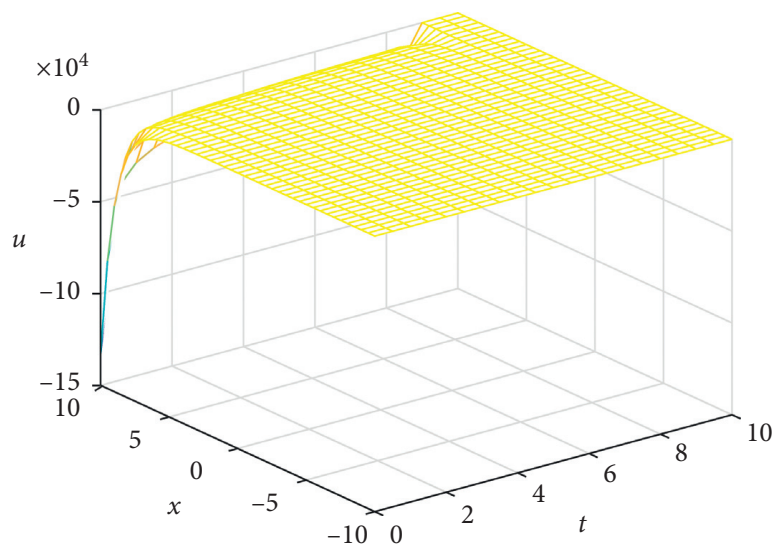

(a)

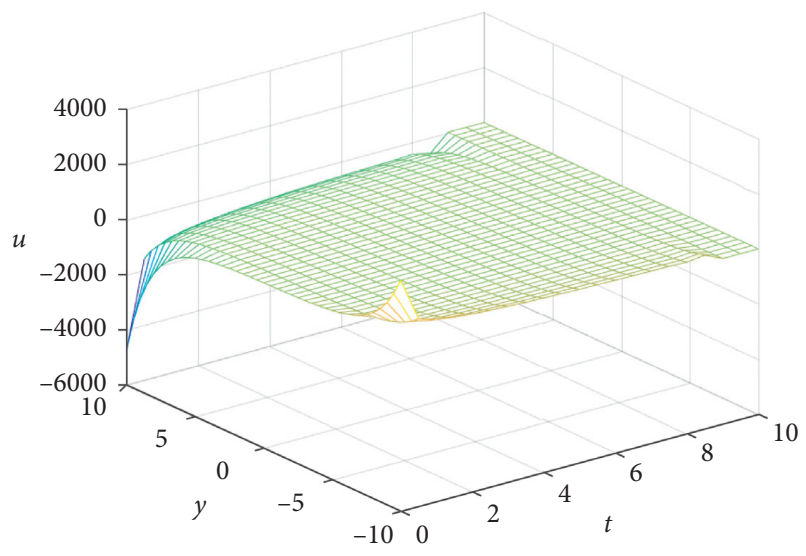

(b)

FIGURE 2: Dynamical profiles of solution (35) on (a) the $(x, t, u)$-plane and (b) the $(y, t, u)$-plane.

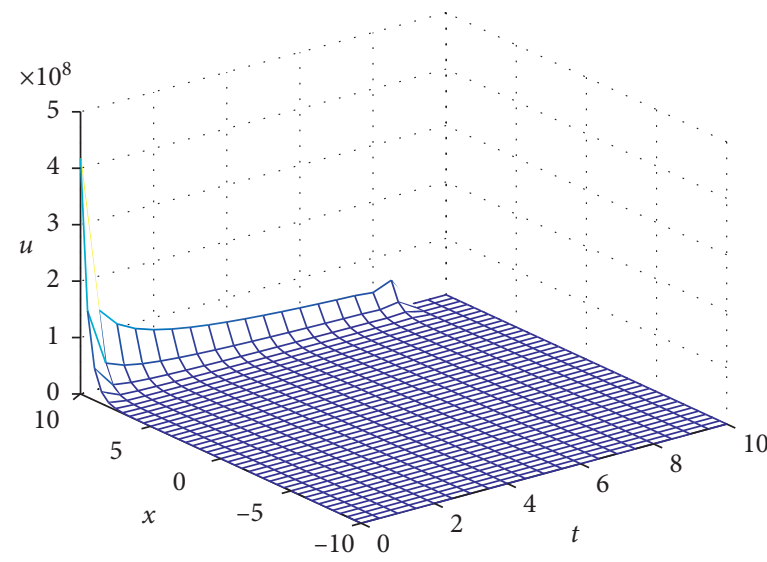

(a)

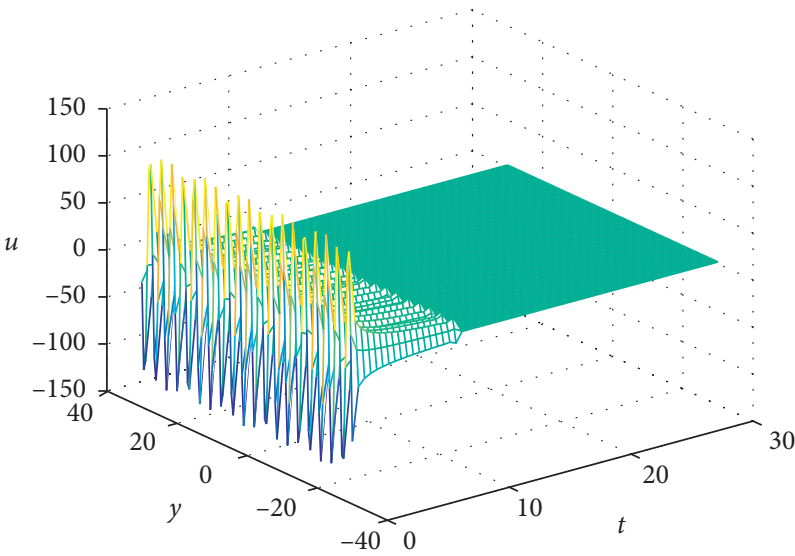

(b)

Figure 3: Dynamical profiles of solution (36) on (a) the $(x, t, u)$-plane and (b) the $(y, t, u)$-plane.

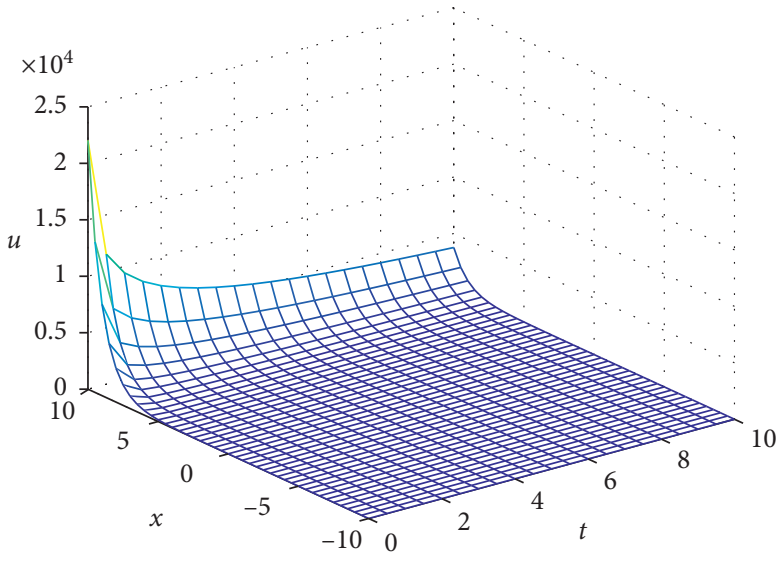

(a)

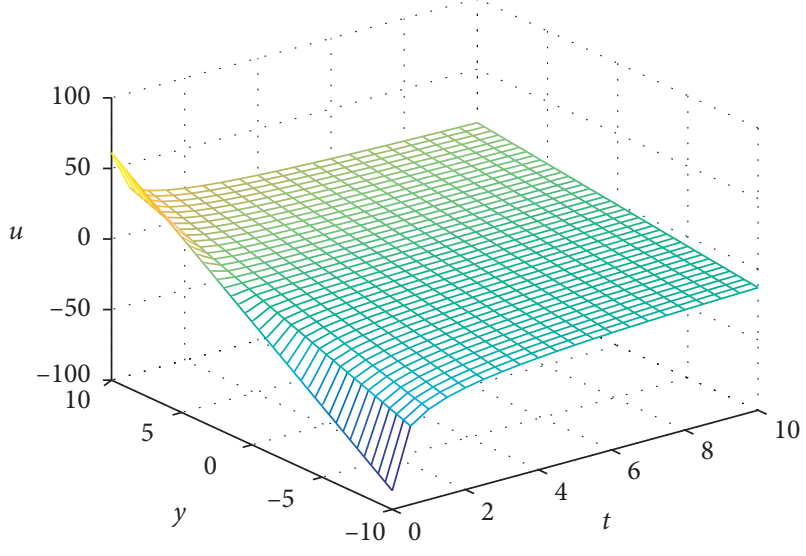

(b)

Figure 4: Dynamical profiles of solution (37) on (a) the $(x, t, u)$-plane and (b) the $(y, t, u)$-plane. 


\subsection{HBP for the $(3+1)$-Dimensional Fractional ZK Equation}

3.2.1. Exact Solutions under Riemann-Liouville Derivatives. The $(3+1)$-dimensional fractional $\mathrm{ZK}$ equation is investigated in this section.

$$
\partial_{t}^{\alpha} u+p u u_{x}+q u_{z z z}+r u_{x x z}+s u_{y y z}=0, \quad 0<\alpha \leq 1,
$$

which represented the acoustic dynamics in a magnetized plasma in three-dimensional space with a low pressure.

The exact solution can be assumed as

$$
u=a_{0} t^{\mu_{1}}+a_{1} v(x, y, z) t^{\mu_{2}} .
$$

Balancing the coefficients of $t$-power by plugging (42) into (41) yields the following two cases:

Cases I. $\mu_{1}-\alpha=\mu_{1}+\mu_{2}=2 \mu_{2}$

That is,

$$
\mu_{1}=\mu_{2}=-\alpha
$$

By comparing the coefficients of $t^{-\alpha}$ and $t^{-2 \alpha}$, we have

$$
\begin{gathered}
\Omega_{0}+p a_{1} v_{x}=0, \quad \Omega_{0}=\frac{\Gamma(1-\alpha)}{\Gamma(1-2 \alpha)}, \\
q v_{z z z}+r v_{x x z}+s v_{y y z}=0 .
\end{gathered}
$$

By solving (44a) directly, we obtain

$$
v(x, y, z)=-\frac{\Omega_{0}}{p a_{1}} x+\phi(y, z) .
$$

Substituting (45) into (44b) yields

$$
q \phi_{z z z}+s \phi_{y y z}=0
$$

Subcase I. Assume $\eta=b_{1} y+b_{2} z+b_{0}$, then we have

$$
\left(q b_{2}^{2}+s b_{1}^{2}\right) \phi^{\prime \prime \prime}(\eta)=0 \text {. }
$$

Solving (47) leads to the following two solutions:

(i) When $q b_{2}^{2}+s b_{1}^{2}=0,(s q<0)$, then

$$
u(x, y, z, t)=\left[a_{0}-\frac{\Gamma(1-\alpha)}{p \Gamma(1-2 \alpha)} x+\phi\left(b_{1} y+b_{2} z+b_{0}\right)\right] t^{-\alpha}, \quad \alpha \neq \frac{1}{2},
$$

where $a_{0}$ and $b_{0}$ are the arbitrary constants and $\phi$ is an arbitrary function.

(ii) When $\phi=c_{2}\left(\eta^{2} / 2\right)+c_{1} \eta+c_{0}$, then

$$
u(x, y, z, t)=\left[a-\frac{\Gamma(1-\alpha)}{p \Gamma(1-2 \alpha)} x+c_{2} \frac{\left(b_{1} y+b_{2} z+b_{0}\right)^{2}}{2}+c_{1}\left(b_{1} y+b_{2} z\right)\right] t^{-\alpha}, \quad \alpha \neq \frac{1}{2},
$$

where $a=a_{0}+c_{0}+c_{1} b_{0}, c_{1}$, and $c_{2}$ are the arbitrary which gives rise to exact solution when $s q<0$ : constants.

Subcase II. Note that (46) is a linear equation, and if $s q<0$, by the linear PDE method, we have

$$
\begin{aligned}
\phi(y, z)= & f_{1}(y)-\frac{2 \sqrt{-s q}}{s} f_{1}\left(\frac{y}{2}-\frac{s z}{2 \sqrt{-s q}}\right) \\
& +f_{2}\left(\frac{\sqrt{-s q}}{s} y+z\right),
\end{aligned}
$$

$$
u(x, y, z, t)=\left[a_{0}-\frac{\Gamma(1-\alpha)}{p \Gamma(1-2 \alpha)} x+a_{1}\left(f_{1}(y)-\frac{2 \sqrt{-s q}}{s} f_{1}\left(\frac{y}{2}-\frac{s z}{2 \sqrt{-s q}}\right)+f_{2}\left(\frac{\sqrt{-s q}}{s} y+z\right)\right)\right] t^{-\alpha}, \quad \alpha \neq \frac{1}{2}
$$

where $a_{0}$ and $a_{1}$ are the arbitrary constants and $f_{1}$ and $f_{2}$ are the two arbitrary functions.

Subcase III. According to the linear PDE method, we assume the solution of (46) as a product form of a separate variable $\phi(y, z)=f(y) g(z)$, thus

$$
q g^{\prime \prime \prime}(z)=c_{0} g^{\prime}(z), s f^{\prime \prime}(y)+c_{0} f(y)=0,
$$

where $c_{0}$ is an arbitrary constant. 
By solving them directly, we obtain the following four solutions (67)-(70) where $a_{0}, a_{1}, c_{1}, c_{2}, c_{3}, c_{4}$, and $c_{5}$ are the arbitrary constants and $\alpha \neq(1 / 2)$ : (i) $\quad \phi=\left(c_{1} \sin \left(\sqrt{\left(c_{0} / s\right)} y\right)\right)+\quad\left(c_{2} \cos \left(\sqrt{\left(c_{0} / s\right)} y\right)\right.$ $\left.\left(c_{3}+c_{4} \sin \left(\sqrt{-\left(c_{0} / q\right)} z\right)+c_{5} \cos \left(\sqrt{-\left(c_{0} / q\right)} z\right)\right)\right)$,

$$
u(x, y, z, t)=\left[a_{0}-\frac{\Gamma(1-\alpha)}{p \Gamma(1-2 \alpha)} x+a_{1}\left(c_{1} \sin \left(\sqrt{\frac{c_{0}}{s}} y\right)+c_{2} \cos \left(\sqrt{\frac{c_{0}}{s}} y\right)\right)\left(c_{3}+c_{4} \sin \left(-\sqrt{\frac{c_{0}}{q}} z\right)+c_{5} \cos \left(-\sqrt{\frac{c_{0}}{q}} z\right)\right)\right] t^{-\alpha} .
$$

(ii) $\quad \phi=\left(c_{1} \sin \left(\sqrt{\left(c_{0} / s\right)} y\right)+c_{2} \cos \left(\sqrt{\left(c_{0} / s\right)} y\right)\right.$

$\left.\left(c_{3}+c_{4} e^{\sqrt{\left(c_{0} / q\right)} z}+c_{5} e^{-\sqrt{\left(c_{0} / q\right)} z}\right)\right)$,

$u(x, y, z, t)=\left[a_{0}-\frac{\Gamma(1-\alpha)}{p \Gamma(1-2 \alpha)} x+a_{1}\left(c_{1} \sin \left(\sqrt{\frac{c_{0}}{s}} y\right)+c_{2} \cos \left(\sqrt{\frac{c_{0}}{s}} y\right)\right)\left(c_{3}+c_{4} e^{\sqrt{\left(c_{0} / q\right)} z}+c_{5} e^{-\sqrt{\left(c_{0} / q\right)} z}\right)\right] t^{-\alpha}$.

(iii) $\phi=\left(c_{1} e^{\sqrt{-\left(c_{0} / s\right)} y}+c_{2} e^{-\sqrt{-\left(c_{0} / s\right)} y}\right)$

$\left(c_{3}+c_{4} \sin \left(\sqrt{-\left(c_{0} / q\right)} z\right)+c_{5} \cos \left(\sqrt{-\left(c_{0} / q\right)} z\right)\right)$,

$u(x, y, z, t)=\left[a_{0}-\frac{\Gamma(1-\alpha)}{p \Gamma(1-2 \alpha)} x+a_{1}\left(c_{1} e^{\sqrt{-\left(c_{0} / s\right)} y}+c_{2} e^{-\sqrt{-\left(c_{0} / s\right)} y}\right)\left(c_{3}+c_{4} \sin \left(\sqrt{-\frac{c_{0}}{q}} z\right)\right)+c_{5} \cos \left(\sqrt{-\frac{c_{0}}{q}} z\right)\right] t^{-\alpha}$.

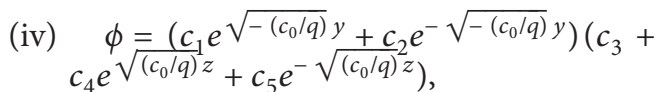

$$
u(x, y, z, t)=\left[a_{0}-\frac{\Gamma(1-\alpha)}{p \Gamma(1-2 \alpha)} x+a_{1}\left(c_{1} e^{\sqrt{-\left(c_{0} / s\right)} y}+c_{2} e^{-\sqrt{-\left(c_{0} / s\right)} y}\right)\left(c_{3}+c_{4} e^{\sqrt{\left(c_{0} / q\right)} z}+c_{5} e^{-\sqrt{\left(c_{0} / q\right)} z}\right)\right] t^{-\alpha} .
$$

Subcase IV. We can also assume the solution of (47) as a summary form of a separate variable $\phi(y, z)=f(y)+g(z)$, then

$$
g^{\prime \prime \prime}(z)=0 .
$$

Thus,

$$
g(z)=\frac{c_{2}^{2} z^{2}}{2}+c_{1} z+c_{0}
$$

which leads to the exact solution by rewriting $b_{2}=a_{1} c_{2}^{2}, b_{1}=a_{1} c_{1}$ and $a=a_{0}+a_{1} c_{0}$ :

$u(x, y, z, t)=\left(a-\frac{\Gamma(1-\alpha)}{p \Gamma(1-2 \alpha)} x+a_{1} f(y)+\frac{b_{2} z^{2}}{2}+b_{1} z\right) t^{-\alpha}, \quad \alpha \neq \frac{1}{2}$,

where $a, a_{1}, b_{2}$, and $b_{1}$ are the arbitrary constants and $f(y)$ is an arbitrary function.
Cases II. $\mu_{2}-\alpha=2 \mu_{2}$ and $\mu_{1}-\alpha=\mu_{1}+\mu_{2}$.

By balancing equation (41), we have

$$
\begin{aligned}
& \mu_{1}=0, \\
& \mu_{2}=-\alpha .
\end{aligned}
$$

Comparing the coefficients of $t^{-\alpha}$ and $t^{-2 \alpha}$ yields

$$
\begin{aligned}
& a_{0} \omega_{0}+p a_{0} a_{1} v_{x}+q a_{1} v_{z z z}+r a_{1} v_{x x z}+s a_{1} v_{y y z}=0 \\
& \omega_{0}=\frac{1}{\Gamma(1-\alpha)} \\
& \Omega_{0} v+p a_{1} v v_{x}=0, \quad \Omega_{0}=\frac{\Gamma(1-\alpha)}{\Gamma(1-2 \alpha)}
\end{aligned}
$$

Solving (61b) leads to the following form: 


$$
v=-\frac{\Omega_{0}}{p a_{1}} x+\phi(y, z)
$$

Substituting (62) into (61a) leads to

$$
q a_{1} \phi_{z z z}+s a_{1} \phi_{y y z}=a_{0}\left(\Omega_{0}-\omega_{0}\right) .
$$

(i) Considering the complex transformation $\eta=b_{1} y+b_{2} z+b_{0}$, we obtain

$$
a_{1} b_{2}\left(q b_{2}^{2}+s b_{1}^{2}\right) \phi^{\prime \prime \prime}(\eta)=a_{0}\left(\Omega_{0}-\omega_{0}\right) .
$$

Solving (81a) and (81b) leads to

$$
\phi(\eta)=\frac{a_{0}\left(\Omega_{0}-\omega_{0}\right)}{6 a_{1} b_{2}\left(q b_{2}^{2}+s b_{1}^{2}\right)} \eta^{3}+\frac{c_{2}}{2} \eta^{2}+c_{1} \eta+c_{0} .
$$

Then, we obtain the exact solution

$$
\begin{aligned}
u(t, x, y, z)= & a_{0}+\left[-\frac{\Gamma(1-\alpha)}{p \Gamma(1-2 \alpha)} x+\frac{a_{0}((\Gamma(1-\alpha) / \Gamma(1-2 \alpha))-(1 / \Gamma(1-\alpha)))}{6 b_{2}\left(q b_{2}^{2}+s b_{1}^{2}\right)}\left(b_{1} y+b_{2} z+b_{0}\right)^{3}+\frac{a_{1} c_{2}}{2}\left(b_{1} y+b_{2} z+b_{0}\right)^{2}\right. \\
& \left.+a_{1} c_{1}\left(b_{1} y+b_{2} z+b_{0}\right)+a_{1} c_{0}\right] t^{-\alpha}, \quad \alpha \neq \frac{1}{2},
\end{aligned}
$$

where $a_{0}, b_{1}, b_{2}, a_{1}, c_{0}, c_{1}$, and $c_{2}$ are the arbitrary constants. (ii) Note that (63) is a linear equation, and by the linear PDE method, we have

$\phi(y, z)=f_{1}(y)+\frac{12 f_{2}((\sqrt{-s q} / s) y+z) s q a_{1}-24 f_{1}((y / 2)-(s z / 2 \sqrt{-s q})) \sqrt{-s q} q a_{1}+a_{0}\left(\Omega_{0}-\omega_{0}\right) s z^{3}+3 q a_{0}\left(\Omega_{0}-\omega_{0}\right) z y^{2}}{12 s q a_{1}}$,

which leads to the exact solution

$u(t, x, y, z)=a_{0}+\left(-\frac{\Omega_{0}}{p} x+a_{1} f_{1}(y)+\frac{12 f_{2}((\sqrt{-s q} / s) y+z) s q a_{1}-24 f_{1}((y / 2)-(s z / 2 \sqrt{-s q})) \sqrt{-s q q a_{1}}+a_{0}\left(\Omega_{0}-\omega_{0}\right) s z^{3}+3 q a_{0}\left(\Omega_{0}-\omega_{0}\right) z y^{2}}{12 s q}\right) t^{-\alpha}, \quad \alpha \neq \frac{1}{2}$,

where $a_{0}$ and $a_{1}$ are constants and $f_{1}$ and $f_{2}$ are the two arbitrary functions.

(iii) Using summary form of the separate variable $\phi=$ $f(y)+g(z)$ yields

$$
q a_{1} g^{\prime \prime \prime}(z)=a_{0}\left(\Omega_{0}-\omega_{0}\right) .
$$

Solving (69) directly, we arrive at

$$
g(z)=\frac{a_{0}\left(\Omega_{0}-\omega_{0}\right)}{6 q a_{1}} z^{3}+\frac{b_{2}}{2} z^{2}+b_{1} z+b_{0},
$$

which gives rise to the exact solution

$u(t, x, y, z)=a_{0}+\left(-\frac{\Gamma(1-\alpha)}{p \Gamma(1-2 \alpha)} x+a_{1} f(y)+\frac{a_{0}}{6 q}\left(\frac{\Gamma(1-\alpha)}{\Gamma(1-2 \alpha)}-\frac{1}{\Gamma(1-\alpha)}\right) z^{3}+a_{1} \frac{b_{2}}{2} z^{2}+a_{1} b_{1} z+a_{1} b_{0}\right) t^{-\alpha}, \quad \alpha \neq \frac{1}{2}$,

where $a_{0}, a_{1}, b_{2}, b_{1}$, and $b_{0}$ are the arbitrary constants and $f(y)$ is an arbitrary function.

(iv) When $a_{0}=0$, using the product form of the separate variable $\phi=f(y) g(z)$ yields

$$
q f(y) g^{\prime \prime \prime}(z)+s f^{\prime \prime \prime}(y) g(z)=0,
$$

which give rises to

$$
f^{\prime \prime}(y)=c_{0} f(y), q g^{\prime \prime \prime}(z)+s c_{0} g(z)=0 .
$$

From (73), we have 


$$
g(z)=c_{1} e^{\left(\sqrt[3]{-s c_{0} q^{2}} / q\right) z}+e^{\left(\sqrt[3]{-s c_{0} q^{2}} / 2 q\right) z}\left(c_{2} \cos \left(\frac{\sqrt{3} \sqrt[3]{-s c_{0} q^{2}}}{2 q} z\right)+c_{3} \sin \left(\frac{\sqrt{3} \sqrt[3]{-s c_{0} q^{2}}}{2 q} z\right)\right)
$$

and following three solutions (75)-(77) where

(i) If $c_{0}>0$, then $a_{1}, b_{1}, b_{2}, c_{1}, c_{2}$, and $c_{3}$ are constants and $\alpha \neq(1 / 2)$ :

$$
\begin{aligned}
u(t, x, y, z)= & \left\{-\frac{\Gamma(1-\alpha)}{p a_{1} \Gamma(1-2 \alpha)} x+\left(b_{1} e^{-\sqrt{c_{0}} y}+b_{2} e^{\sqrt{c_{0}} y}\right) c_{1} e^{\left(\sqrt[3]{-s c_{0} q^{2}} / q\right) z}+e^{\left(\sqrt[3]{-s c_{0} q^{2}} / 2 q\right) z}\left(c_{2} \cos \left(\frac{\sqrt{3} \sqrt[3]{-s c_{0} q^{2}}}{2 q} z\right)\right.\right. \\
& \left.\left.+c_{3} \sin \left(\frac{\sqrt{3} \sqrt[3]{-s c_{0} q^{2}}}{2 q} z\right)\right)\right\} t^{-\alpha} .
\end{aligned}
$$

(ii) If $c_{0}<0$, then

$$
\begin{aligned}
u(t, x, y, z)= & \left\{-\frac{\Gamma(1-\alpha)}{p a_{1} \Gamma(1-2 \alpha)} x+\left(b_{1} \cos \left(\sqrt{-c_{0} y}\right)+b_{2} \sin \left(\sqrt{-c_{0} y}\right)\left[c_{1} e^{\left(\sqrt[3]{-s c_{0} q^{2}} / q\right) z}+e^{\left(\sqrt [ 3 ] { - s c _ { 0 } q ^ { 2 } / 2 q ) z } \left(c_{2} \cos \left(\frac{\sqrt{3} \sqrt[3]{-s c_{0} q^{2}}}{2 q} z\right)\right.\right.}\right.\right.\right. \\
& \left.\left.\left.+c_{3} \sin \left(\frac{\sqrt{3} \sqrt[3]{-s c_{0} q^{2}}}{2 q} z\right)\right)\right]\right\} t^{-\alpha} .
\end{aligned}
$$

(iii) If $c_{0}=0$, then

$$
u(t, x, y, z)=\left(-\frac{\Gamma(1-\alpha)}{p a_{1} \Gamma(1-2 \alpha)} x+\left(b_{1} y+b_{2}\right)\left(c_{1} z^{2}+c_{2} z+c_{3}\right)\right) t^{-\alpha}
$$

Remark 5. In case I, we do not concern the complex transform $\xi=a x+b y+c z+d$ for (48) contains solutions of this type.

Remark 6. In case II, we do not consider the complex transform $\xi=a x+b y+c z+d$ for omitting the trivial solution.

Remark 7 . The singularity only appears when $\alpha=(1 / 2)$ in solutions (53)-(56), (66), (68), (71), and (76)-(78) in the Riemann-Liouville case.

Remark 8. If taking form (10) into the fractional ZK model (41), we see that the nonlinear term $u u_{x}$ will lead to trivial solutions, thus the Caputo derivative is not considered.

\subsubsection{Dynamical Analysis of Exact Solution for Fractional ZK Model}

(i) As can be seen from Figure 5, taking $\alpha=(3 / 5), a=$ $3, a_{0}=c_{0}=1, c_{1}=\quad(1 / 2), b_{0}=2, b_{1}=1, b_{2}=-2$, $c_{2}=2, t \in[0,10], y=1.5, z=2.5, x \in[-10,10]$, $x=-2.5, z=2.5, y \in[-10,10]$, and $x=-2.5, y=$
1.5, $z \in[-10,10]$, it is shown that solution (49) increases with increase in spatial variables and decreases as time increases. When $x, y$, and $z$ are fixed, solution (49) tends to 0 at $\alpha$ decay rate as $t \longrightarrow \infty$, the larger the $\alpha$ is, the faster the $u$ decay is.

(ii) From Figure 6, by taking $\alpha=(2 / 5), a_{0}=(1 / 2), a_{1}=$ $2, c_{1}=1.5, c_{2}=-2, c_{3}=-3, c_{4}=-2.5, c_{5}=1, c_{0}=$ $2, p=3, s=q=1 / 2, t \in[0,30], x=-2.5, z=2.5$, $y \in[-30,30]$ and $t \in[0,10]$, and $x=-2.5, y=$ $1.5, z \in[-10,10]$, we see solution (54) is periodic bounded and tends to 0 at $\alpha$ decay rate as $y$ and $t$ tend to $\infty$ if $x$ and $z$ are fixed. The larger the $\alpha$ is, the faster the $u$ decay is. When $x$ and $y$ are fixed, solution (54) decreases to $-\infty$ as $z$ increases to $+\infty$.

(iii) As can be seen from Figure 7 , selecting $\alpha=(2 / 3), p=3, a_{0}=2, a_{1}=1, b_{1}=2, b_{2}=-1, b_{0}=$ $3, c_{0}=1, c_{1}=(1 / 2), c_{2}=2, q=s=(1 / 2), t \in$ $[0,10], y=1.5, z=2.5, x \in[-10,10], x=-2.5, z=$ $2.5, y \in[-10,10], \quad$ and $\quad x=-2.5, y=1.5, z \in$ $[-10,10]$, while from Figure 8 by choosing $\alpha=(2 / 3), p=3, a_{1}=1, b_{1}=1, b_{2}=-1.5, c_{1}=1.5$, 


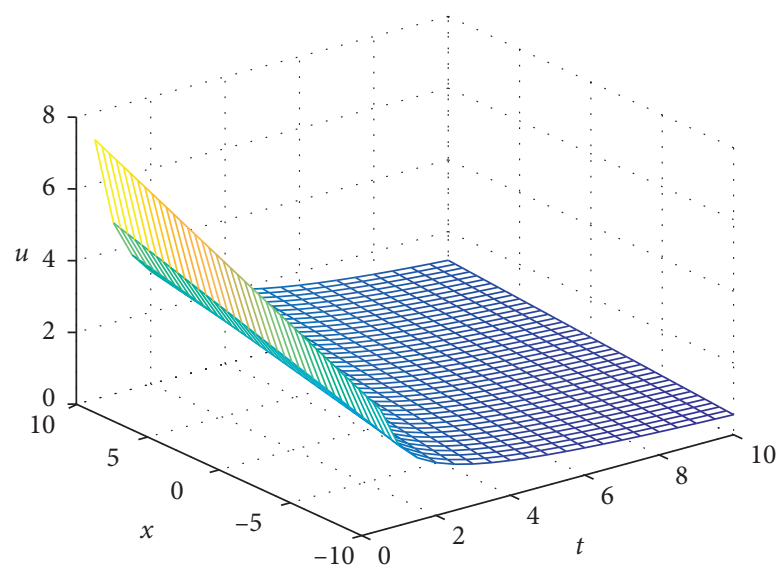

(a)

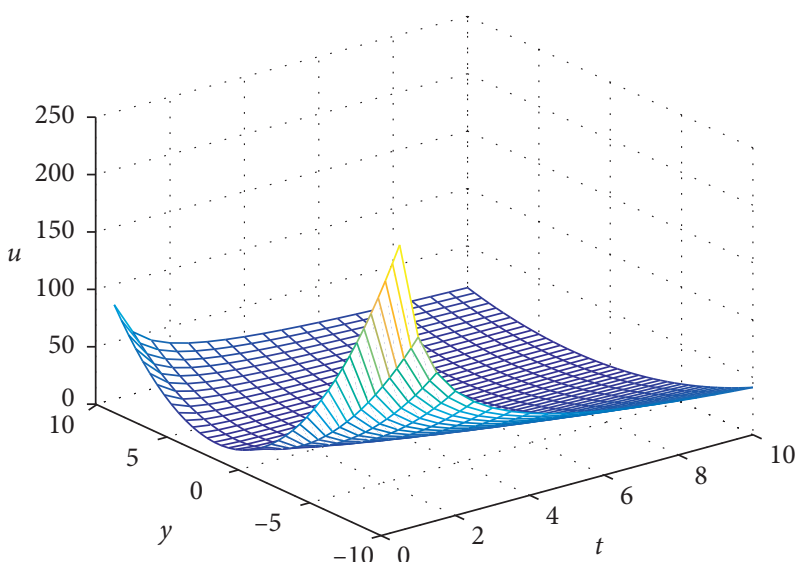

(b)

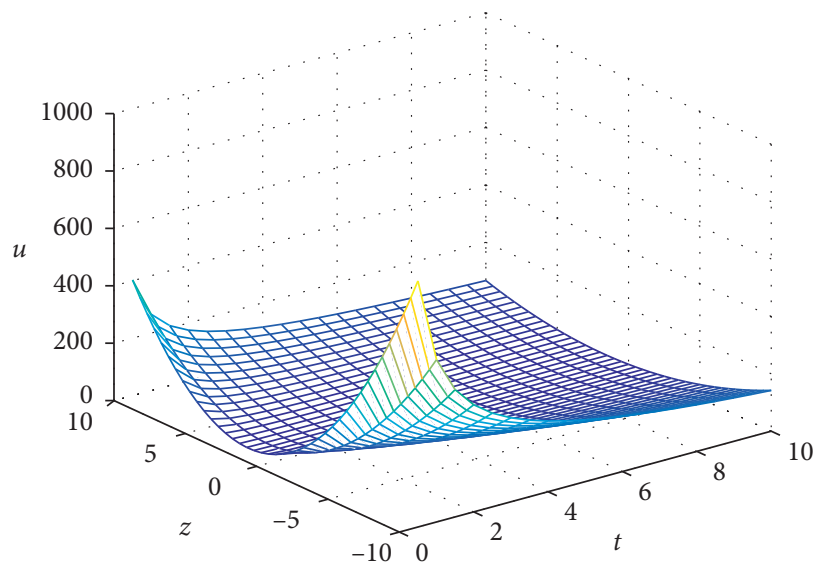

(c)

Figure 5: Dynamical profiles of solution (49) on (a) the $(x, t, u)$-plane, (b) the $(y, t, u)$-plane, and (c) the $(z, t, u)$-plane.

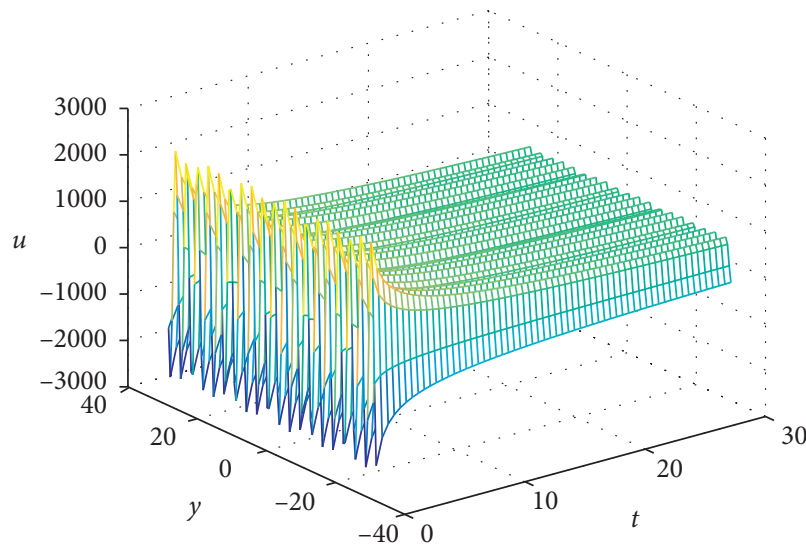

(a)

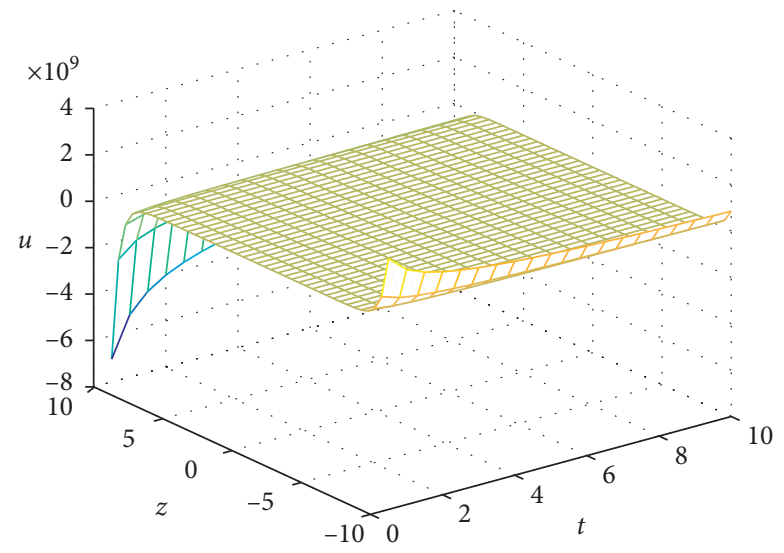

(b)

Figure 6: Dynamical profiles of solution (54) on (a) the $(y, t, u)$-plane and (b) the $(z, t, u)$-plane.

$c_{2}=2, c_{3}=-2.5, c_{0}=2, s=8, q=2, t \in[0,10]$ $y=1.5, z=2.5, x \in[-10,10], \quad x=-2.5, z=2.5$, $y \in[-10,10]$, and $x=-2.5, y=1.5, z \in[-10,10]$, it is shown that solutions (66) and (75) increase to $\infty$ or decrease to $-\infty$ as $x, y$, or $z$ increases to $\infty$, and ones also tends to 0 at $\alpha$ decay rate as $t \longrightarrow \infty$ if $x, y$, and $z$ are fixed. The larger the $\alpha$ is, the faster the $u$ decay is. 


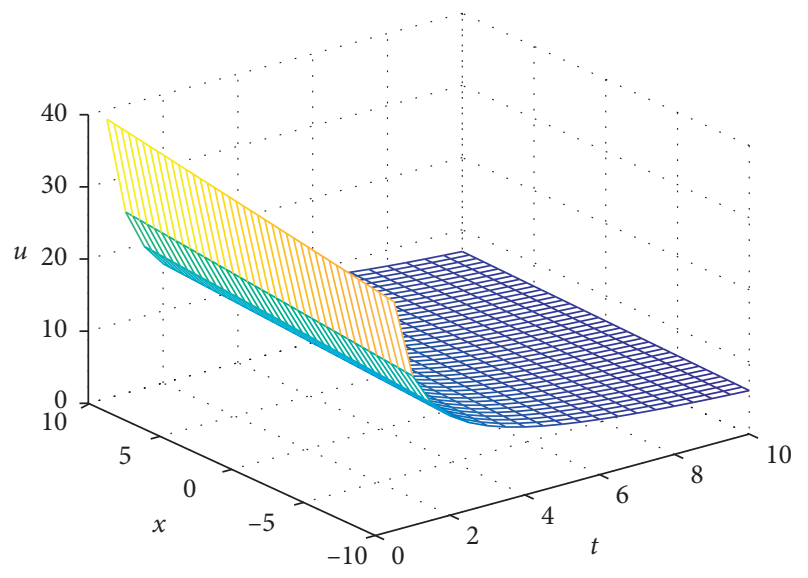

(a)

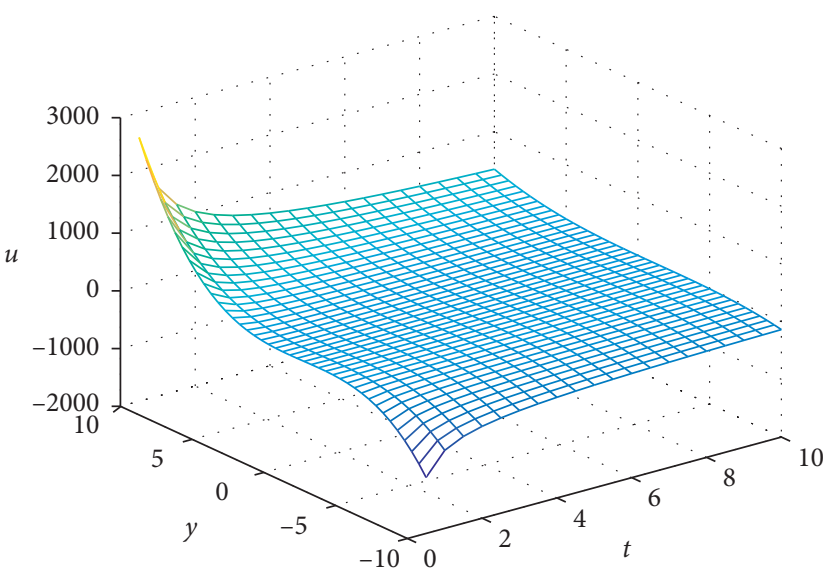

(b)

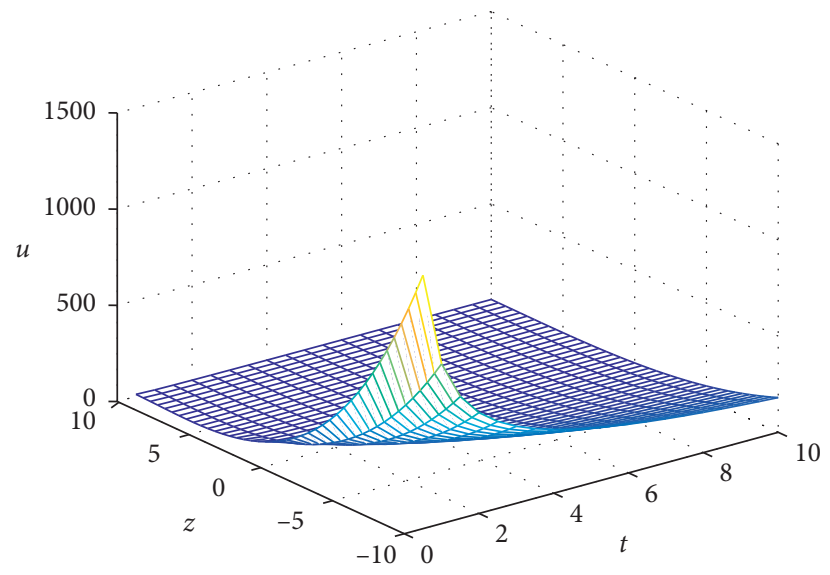

(c)

Figure 7: Dynamical profiles of solution (66) on (a) the $(x, t, u)$-plane, (b) the $(y, t, u)$-plane, and (c) the $(z, t, u)$-plane.

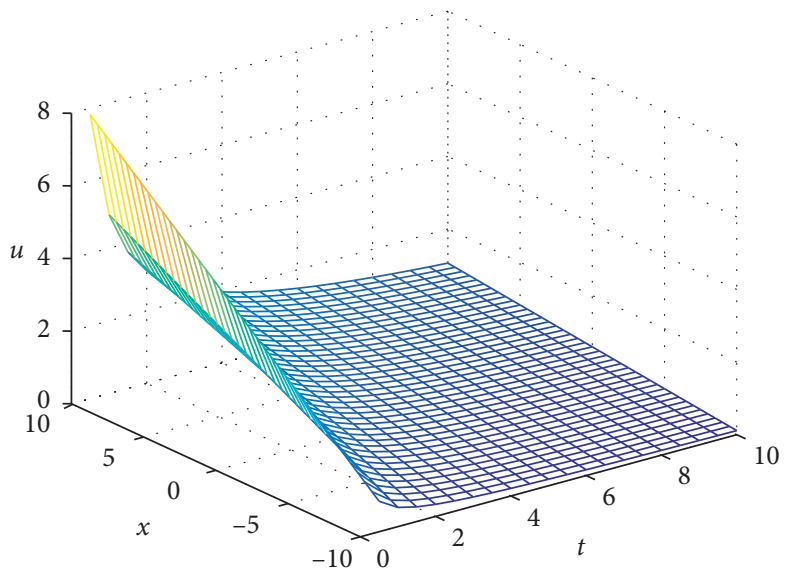

(a)

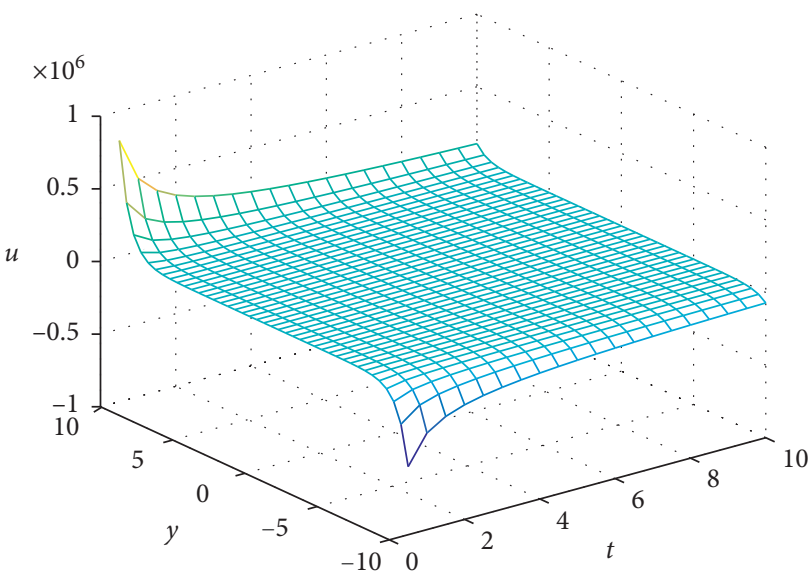

(b)

Figure 8: Continued. 


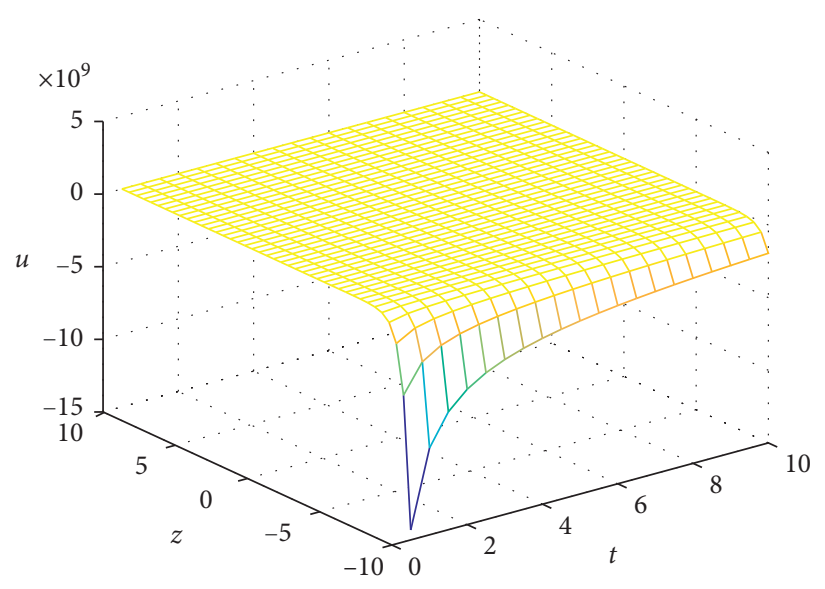

(c)

Figure 8: Dynamical profiles of solution (75) on (a) the $(x, t, u)$-plane, (b) the $(y, t, u)$-plane, and (c) the $(z, t, u)$-plane.

(iv) From Figure 9, as we choose $\alpha=(2 / 3), p=1, a_{1}=$ $3, b_{1}=1, b_{2}=-1.5, c_{0}=2, c_{1}=1, c_{2}=2, c_{3}=-2.5$, $s=8, q=2, x=z=-2.5, y \in[-30,30], t \in[0,30]$, the solution (76) is periodic bounded and has $\alpha$ decay rate as $t \longrightarrow \infty$ if $x$ and $z$ are fixed, and one has the same dynamical properties as (54) if else.

3.3. HBP for the $(3+1)$-Dimensional Fractional JM Equation. The $(3+1)$-dimensional fractional JM equation will be researched in the following contents:

$$
u_{x x x y}+p u_{y} u_{x x}+q u_{x} u_{x y}+r \partial_{t}^{\alpha} u_{y}-s u_{x z}=0, \quad 0<\alpha \leq 1,
$$

which is developed from the second members of integrable systems of the classical KP hierarchy.

\subsubsection{Exact Solutions under Caputo Derivatives. Assume}

$$
u=a_{0}+a_{1} v(x, y, z) E_{\alpha, 1}\left(\lambda t^{\alpha}\right) .
$$

Injecting (79) into (78), we arrive at

$$
\begin{aligned}
v_{x x x y}+r \lambda v_{y}-s v_{x z} & =0, \\
p v_{y} v_{x x}+q v_{x} v_{x y} & =0 .
\end{aligned}
$$

(i) We assume that $v=v(\xi), \xi=a x+b y+c z+d$, then (80a) and (80b) becomes the following ODEs:

$$
\begin{array}{r}
a^{3} b v^{(4)}(\xi)+r \lambda b v^{\prime}(\xi)-s a c v^{\prime \prime}(\xi)=0, \\
(p+q) v^{\prime}(\xi) v^{\prime \prime}(\xi)=0 .
\end{array}
$$

When $q=-p,(81 \mathrm{~b})$ is a free equation, and then solving (81a), we have following solutions by noting $\Delta=\sqrt[3]{\left(\sqrt{3}\left(\sqrt{27 r^{2} \lambda^{2} b^{3}-4 s^{3} c^{3} / b}\right)-9 r \lambda b\right)} b^{2}$,

$$
\begin{aligned}
v(\xi)= & \frac{c_{0}}{b \lambda r}+c_{1} e^{\left(12^{(1 / 3)} \Delta^{2}+12^{(2 / 3)} s c b / 6 a b \Delta\right) \xi}+e^{-\left(12^{(1 / 3)} \Delta^{2}+12^{(2 / 3)} s c b / 12 a b \Delta\right) \xi}\left[c_{2} \cos \left(\frac{\sqrt{3}\left(12^{(1 / 3)} \Delta^{2}-12^{(2 / 3)} s c b\right)}{12 a b \Delta} \xi\right)\right. \\
& \left.+c_{3} \sin \left(\frac{\sqrt{3}\left(12^{(1 / 3)} \Delta^{2}-12^{(2 / 3)} s c b\right)}{12 a b \Delta} \xi\right)\right]
\end{aligned}
$$

which leads to the exact solution:

$$
\begin{aligned}
u(t, x, y, z)= & a_{0}+a_{1}\left\{\frac{c_{0}}{b \lambda r}+c_{1} e^{\left(12^{(1 / 3)} \Delta^{2}+12^{(2 / 3)} s c b / 6 a b \Delta\right)(a x+b y+c z+d)}+e^{-\left(12^{(1 / 3)} \Delta^{2}+12^{(2 / 3)} s c b / 12 a b \Delta\right)(a x+b y+c z+d)}\right. \\
& \cdot\left[c _ { 2 } \operatorname { c o s } \left(\frac{\sqrt{3}\left(12^{(1 / 3)} \Delta^{2}-12^{(2 / 3)} s c b\right)}{12 a b \Delta}\right.\right. \\
& \left.\left.\cdot(a x+b y+c z+d))+c_{3} \sin \left(\frac{\sqrt{3}\left(12^{(1 / 3)} \Delta^{2}-12^{(2 / 3)} s c b\right)}{12 a b \Delta}(a x+b y+c z+d)\right)\right]\right\} E_{\alpha, 1}\left(\lambda t^{\alpha}\right),
\end{aligned}
$$




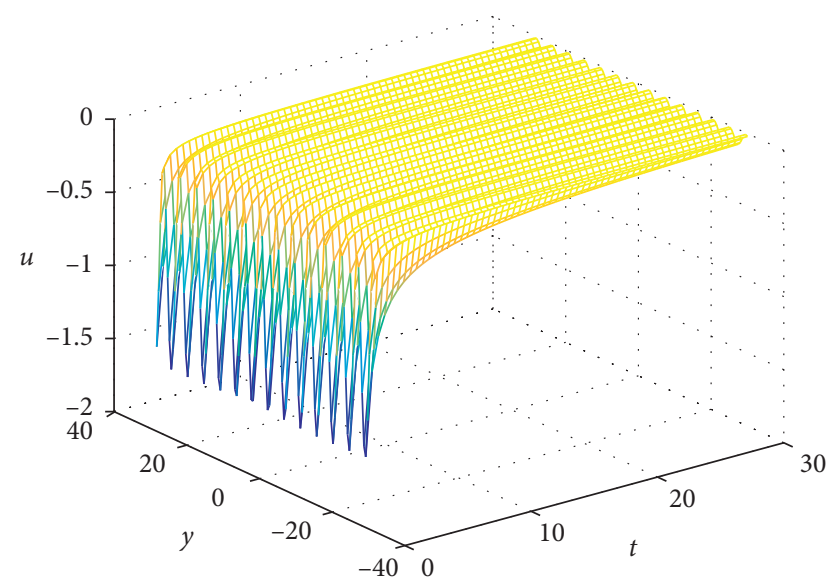

Figure 9: Dynamical profiles of solution $(76)$ on the $(y, t, u)$-plane.

where $a_{0}, a_{1}, c_{0}, a, b, c$, and $d$ are the arbitrary constants.

Remark 9. In case of $q \neq-p$, from $v^{\prime \prime}(\xi)=0$, we obtain $v^{\prime}(\xi)=0$ which only leads to a trivial solution, thus we do not consider this case.

(ii) Assume $v(x, y, z)=v(\eta, z),(\eta=a x+b y+c)$, and from (80a) and (80b), we have

$$
\begin{aligned}
a^{3} b v_{\eta \eta \eta \eta}+r \lambda b v_{\eta}-s a v_{\eta z} & =0, \\
(p+q) v_{\eta} v_{\eta \eta} & =0 .
\end{aligned}
$$

Then (84a) and (84b) yields the following two subcases (1) If $q=-p$, we only consider (84a).

(i) Note that it is a linear equation, and we suppose $v(\eta, z)=f(\eta) g(z)$, which arrives at

$$
a^{3} b f^{(4)}(\eta) g(z)+r \lambda b f^{\prime}(\eta) g(z)-s a f^{\prime}(\eta) g^{\prime}(z)=0 \text {. }
$$

Using the separate variable method, we have

$$
\begin{aligned}
g^{\prime}(z) & =c g(z), \\
a^{3} b f^{\prime \prime \prime}(\eta)+(r \lambda b-s a c) f(\eta) & =c_{0} .
\end{aligned}
$$

Solving (86a) reads that

$$
g(z)=b_{0} e^{c z}
$$

and the solution of (86b) and the exact solution of (78) is described as the following two cases:

If $r \lambda b \neq s a c$, then

$$
f(\eta)=\frac{c_{0}}{r b \lambda-s a c}+c_{1} e^{\left(\sqrt[3]{(s a c-r b \lambda) b^{2}} / a b\right) \eta}+e^{-\left(\sqrt[3]{(s a c-r b \lambda) b^{2} / 2 a b}\right)} \eta\left[c_{2} \cos \left(\frac{\sqrt{3} \sqrt[3]{(s a c-r b \lambda) b^{2}}}{2 a b} \eta\right)+c_{3} \sin \left(\frac{\sqrt{3} \sqrt[3]{(s a c-r b \lambda) b^{2}}}{2 a b} \eta\right)\right]
$$

and the exact solution is

$$
\begin{aligned}
& u(t, x, y, z)=a_{0}+a_{1} b_{0}\left\{\frac{c_{0}}{r b \lambda-s a c}+c_{1} e^{\left(\sqrt[3]{(s a c-r b \lambda) b^{2}} / a b\right)(a x+b y+c)}+e^{-\left(\sqrt[3]{(s a c-r b \lambda) b^{2}} / 2 a b\right)(a x+b y+c)}\right. \\
& \cdot\left[c_{2}\left(\cos \left(\frac{\sqrt{3} \sqrt[3]{(s a c-r b \lambda) b^{2}}}{2 a b} x+b y+c\right)\right)\right. \\
& \left.\cdot\left(a+c_{3} \sin \left(\frac{\sqrt{3} \sqrt[3]{(s a c-r b \lambda) b^{2}}}{2 a b}(a x+b y+c)\right)\right) e^{c z}\right\} E_{\alpha, 1}\left(\lambda t^{\alpha}\right), \\
& f(\eta)=\frac{c_{0}}{6} \eta^{3}+\frac{c_{2}}{2} \eta^{2}+c_{1} \eta+c_{3}
\end{aligned}
$$
trary constants.

If $c=(r b \lambda / s a)$, then and the exact solution is

$$
u(t, x, y, z)=a_{0}+a\left(\frac{c_{0}}{6}\left(a x+b y+\frac{r b \lambda}{s a}\right)^{3}+\frac{c_{2}}{2}\left(a x+b y+\frac{r b \lambda}{s a}\right)^{2}+c_{1}\left(a x+b y+\frac{r b \lambda}{s a}\right)+c_{3}\right) e^{(r b \lambda / s a) z} E_{\alpha, 1}\left(\lambda t^{\alpha}\right)
$$


where $a_{0}, c_{0}, a, b, c_{1}, c_{2}$, and $c_{3}$ are the arbitrary constants. (ii) Solving (84a) directly by the linear PDE method reads that

$$
\begin{aligned}
v(\eta, z)= & c^{-(1 / 3)}\left[c_{1} e^{c^{(1 / 3)} \eta+\left(\left(b a^{2} c / s\right)+(b r \lambda / s a)\right) z}+4 e^{-(1 / 2) c^{(1 / 3)} \eta+\left(\left(b a^{2} c / s\right)+(b r \lambda / s a)\right) z}\left(c_{2} \sin \left(\frac{\sqrt{3} c^{(1 / 3)}}{2} \eta\right)+c_{3} \cos \left(\frac{\sqrt{3} c^{(1 / 3)}}{2} \eta\right)\right)\right]+f(z), \\
u(t, x, y, z)= & a_{0}+a_{1}\left\{c ^ { - ( 1 / 3 ) } \left[c_{1} e^{c^{(1 / 3)}(a x+b y+c)+\left(\left(b a^{2} c / s\right)+(b r \lambda / s a)\right) z}+4 e^{-(1 / 2) c^{(1 / 3)}(a x+b y+c)+\left(\left(b a^{2} c / s\right)+(b r \lambda / s a)\right) z}\right.\right. \\
& \left.\left.\cdot\left(c_{2} \sin \left(\frac{\sqrt{3} c^{(1 / 3)}}{2}(a x+b y+c)\right)+c_{3} \cos \left(\frac{\sqrt{3} c^{(1 / 3)}}{2}(a x+b y+c)\right)\right)\right]+f(z)\right\} E_{\alpha, 1}\left(\lambda t^{\alpha}\right),
\end{aligned}
$$

where $a_{0}, a_{1}, a, b, c, c_{1}, c_{2}$, and $c_{3}$ are the arbitrary constants and $f(z)$ is an arbitrary function.

(iii) Assume $v(\eta, z)=f(\eta)+g(z)$, and from (84a), we have

$$
a^{3} f^{(4)}(\eta)+r \lambda f^{\prime}(\eta)=0
$$

and then

$$
a^{3} f^{\prime \prime \prime}(\eta)+r \lambda f(\eta)=c_{0}
$$

By solving (94), we have

$$
\begin{aligned}
f(\eta)= & \frac{c_{0}}{r \lambda}+c_{1} e^{-(\sqrt[3]{r \lambda} / a) \eta}+e^{(\sqrt[3]{r \lambda} / 2 a) \eta}\left(c_{2} \cos \left(\frac{\sqrt{3} \sqrt[3]{r \lambda}}{2 a} \eta\right)\right. \\
& \left.+c_{3} \sin \left(\frac{\sqrt{3} \sqrt[3]{r \lambda}}{2 a} \eta\right)\right),
\end{aligned}
$$

and the exact solution

$$
\begin{aligned}
u(t, x, y, z)= & a_{0}+a_{1}\left[\frac{c_{0}}{r \lambda}+c_{1} e^{-(\sqrt[3]{r \lambda} / a)(a x+b y+c)}+e^{(\sqrt[3]{r \lambda} / 2 a)(a x+b y+c)}\left(c_{2} \cos \left(\frac{\sqrt{3} \sqrt[3]{r \lambda}}{2 a}(a x+b y+c)\right)\right.\right. \\
& \left.\left.+c_{3} \sin \left(\frac{\sqrt{3} \sqrt[3]{r \lambda}}{2 a}(a x+b y+c)\right)+g(z)\right)\right] E_{\alpha, 1}\left(\lambda t^{\alpha}\right),
\end{aligned}
$$

where $a_{0}, a_{1}, a, b, c, c_{1}, c_{2}$, and $c_{3}$ are the arbitrary constants and $g(z)$ is an arbitrary function.

(2) If $q \neq-p$, then for the nontrivial case, we have $v_{\eta \eta}=0$, and this leads to

$$
v=f_{1}(z) \eta+f_{2}(z) .
$$

Then, substituting (97) into (84a), we have

$$
r \lambda b f_{1}(z)-s a f_{1}^{\prime}(z)=0
$$

which yields

$$
f_{1}(z)=c_{0} e^{(r \lambda b / s a) z}
$$

This gives rise to the exact solution

$$
\begin{aligned}
u(t, x, y, z)= & a_{0}+a_{1}\left(c_{0} e^{(r \lambda b / s a) z}(a x+b y+c)\right. \\
& \left.+f_{2}(z)\right) E_{\alpha, 1}\left(\lambda t^{\alpha}\right),
\end{aligned}
$$

where $a_{0}, a_{1}, c_{0}, a, b$, and $c$ are the arbitrary constants and $f_{2}(z)$ is an arbitrary function.
(III) Assume $v=f(x, z)+g(y, z)$.

Then, from (84a) and (84b), we arrive at

$$
\begin{array}{r}
g_{y} f_{x x}=0, \\
r \lambda g_{y}-s f_{x z}=0 .
\end{array}
$$

For the nontrivial case, we obtain

$$
f_{x x}=0 \Rightarrow f=f_{1}(z) x+f_{2}(z) .
$$

Injecting (102) into (101b) yields

$$
g=\frac{s}{r \lambda} f_{1}^{\prime}(z) y+g_{0}(z)
$$

which leads to the exact solution

$u(t, x, y, z)=a_{0}+a_{1}\left(f_{1}(z) x+\frac{s}{r \lambda} f_{1}^{\prime}(z) y+\psi(z)\right) E_{\alpha, 1}\left(\lambda t^{\alpha}\right)$,

where $a_{0}$ and $a_{1}$ are the arbitrary constants and $f_{1}(z)$ and $\psi(z)$ are the two arbitrary functions. 
3.3.2. Exact Solutions under Riemann-Liouville Derivatives. We assume

$$
u=a_{0} t^{\mu_{1}}+a_{1} v(x, y, z) t^{\mu_{2}}
$$

leads to $\mu_{2}-\alpha=2 \mu_{2}$ and $\mu_{2}=-\alpha$.

Substituting (105) into (78) by comparing the coefficients of $t^{-\alpha}$ and $t^{-2 \alpha}$, we have

$$
\begin{gathered}
a_{1}\left(p v_{y} v_{x x}+q v_{x} v_{x y}\right)+r \Omega_{0} v_{y}=0, \quad \Omega_{0}=\frac{\Gamma(1-\alpha)}{\Gamma(1-2 \alpha)}, \\
v_{x x x y}-s v_{x z}=0 .
\end{gathered}
$$

(I) Note that (106b) is a linear equation, and we assume the summary type of a separate variable $v=f(x, z)+$ $g(y, z)$ which yields

$$
\begin{aligned}
p a_{1} f_{x x}+r \Omega_{0} & =0, \\
f_{x z} & =0 .
\end{aligned}
$$

Then, from (107b), we have

$$
f(x, z)=f_{1}(x)+f_{2}(z) .
$$

Plugging (108) into (107a), we arrive at

$$
f_{1}(x)=-\frac{r \Omega_{0}}{2 p a_{1}} x^{2}+c_{1} x+c_{0}
$$

This gives rise to the exact solution

$$
u(t, x, y, z)=a_{0} t^{\mu_{1}}+\left[-\frac{r \Gamma(1-\alpha)}{2 p \Gamma(1-2 \alpha)} x^{2}+b_{1} x+a_{1} f_{2}(z)+a_{1} g(y, z)+b_{0}\right] t^{-\alpha}, \quad \alpha \neq \frac{1}{2}, \mu_{1} \neq \alpha-1,
$$

where $\mu_{1}, a_{0}, a_{1}, b_{1}=a_{1} c_{1}$ and $b_{0}=a_{1} c_{0}$ are the arbitrary constants and $f_{2}(z)$ and $g(y, z)$ are the two arbitrary functions.

(II) Noticing equation (106a), we assume that $v=v(\xi, z),(\xi=a x+b y+c)$, then (106a) and (106b) become

$$
\begin{gathered}
a_{1} a^{2}(p+q) v_{\xi \xi}+r \Omega_{0}=0, \quad \Omega_{0}=\frac{\Gamma(1-\alpha)}{\Gamma(1-2 \alpha)}, \\
v_{z}=-\frac{\psi(z)}{s a} .
\end{gathered}
$$

Solving (111a) leads to

$$
v=-\frac{r \Omega_{0}}{2 a_{1} a^{2}(p+q)} \xi^{2}+\phi_{1}(z) \xi+\phi_{0}(z)
$$

Substituting (112) into (111b) yields

$$
\phi_{1}^{\prime}(z) \xi+\phi_{0}^{\prime}(z)=-\frac{\psi(z)}{s a} .
$$

By comparing the coefficients, we have

$$
\begin{aligned}
& \phi_{1}(z)=c_{1}, \\
& \phi_{0}(z)=-\frac{1}{s a} \int_{z} \psi(\bar{z}) \mathrm{d} \bar{z}=-\frac{1}{s a} \phi(z),
\end{aligned}
$$

which gives rise to the exact solution

$$
u(t, x, y, z)=a_{0} t^{\mu_{1}}+\left[-\frac{r \Gamma(1-\alpha)}{2 a^{2}(p+q) \Gamma(1-2 \alpha)}(a x+b y+c)^{2}+a_{1} c_{1}(a x+b y+c)-\frac{a_{1}}{s a} \phi(z)\right] t^{-\alpha}, \quad \alpha \neq \frac{1}{2}, \mu_{1} \neq \alpha-1
$$

where $a_{0}, a_{1}, c_{1}, a, b$, and $c$ are the arbitrary constants and $\phi(z)$ is an arbitrary function.

Remark 10. We do not consider the transformation $\xi=a x+b y+c z+d$ for omitting the trivial solution.

Remark 11. The singularity only appears when $\alpha=(1 / 2)$ and $\mu_{1}=\alpha-1$ in solutions (110) and (115) in the Riemann-Liouville case.
3.3.3. Dynamical Analysis of Exact Solutions for Fractional JM Equation. Under the Caputo case:

(i) As can be seen from Figure 10, taking $\alpha=(1 / 2), \mu_{1}=$ $\alpha-1, a_{0}=1, a_{1}=1, c_{0}=1, c_{1}=$ $2, c_{2}=1, c_{3}=-3, a=2, r=b=s=c=1, \lambda=$ $-1, d=-3 y=1.5, z=2, t \in[0,10], x \in[-10,10]$, it is shown that solution (83) increases with increase in the spatial variable and decreases as time increases on all $(u, t, x),(u, t, y)$, and $(u, t, z)$-planes.

(ii) As Figures 11 and 12 show, we see that by choosing $a=b=1, s=(7 / 2), c=2, r=2, \alpha=(1 / 2), \lambda=-(1 / 2), a_{0}=$ 


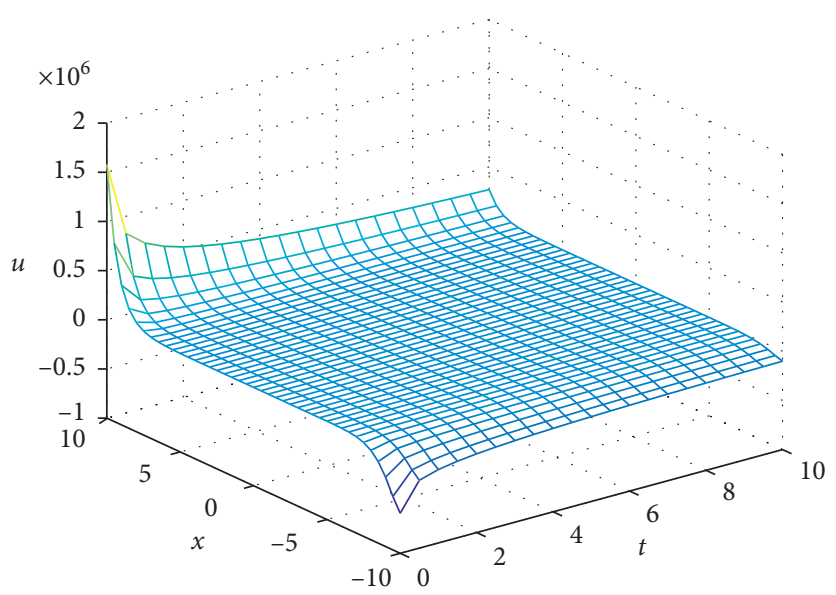

FIgURE 10: Dynamical profiles of solution (83) on the $(x, t, u)$-plane.

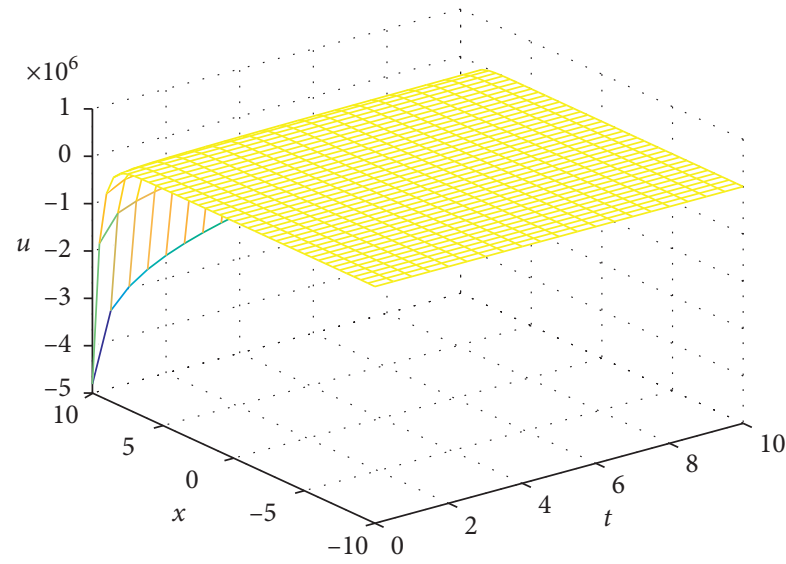

(a)

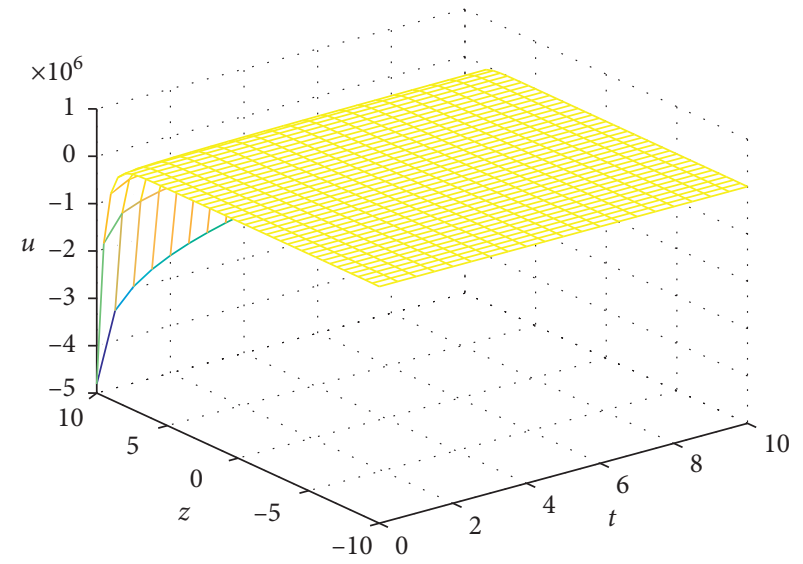

(b)

FIgUre 11: Dynamical profiles of solution (89) on (a) the $(x, t, u)$-plane and (b) the $(z, t, u)$-plane.

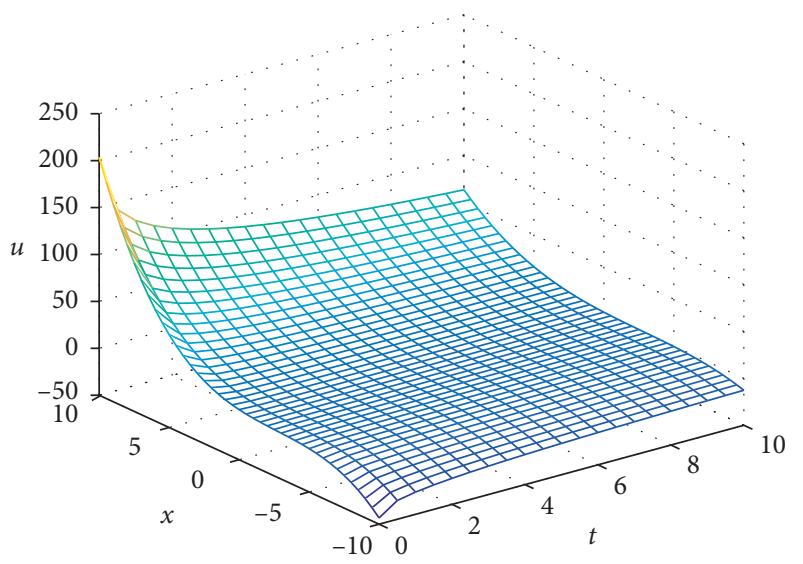

(a)

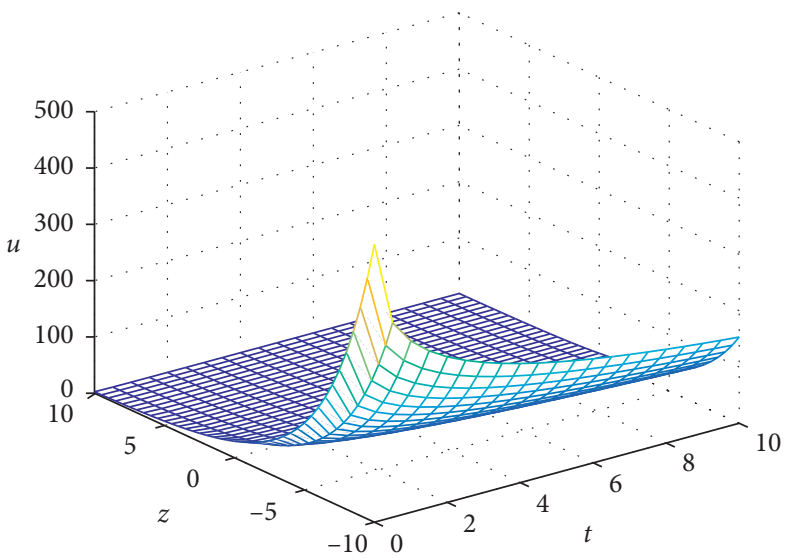

(b)

FIgURe 12: Dynamical profiles of solution (91) on (a) the $(x, t, u)$-plane and (b) the $(z, t, u)$-plane. 


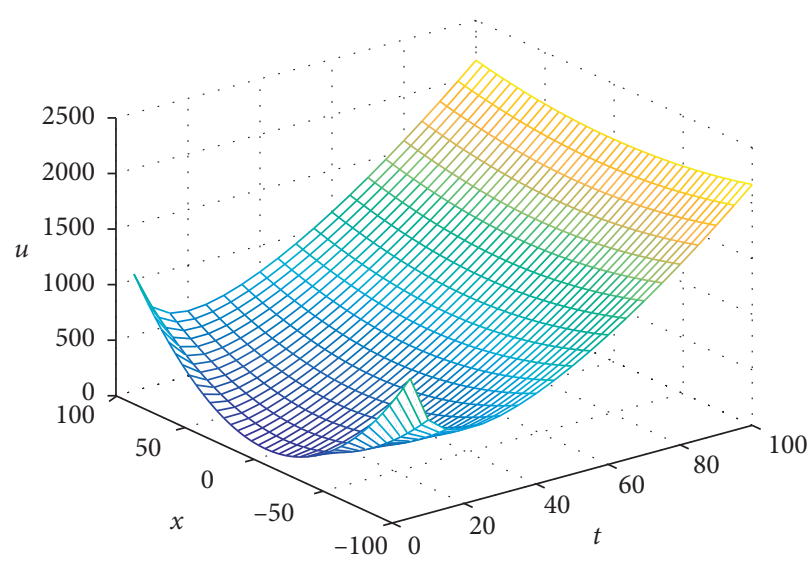

(a)

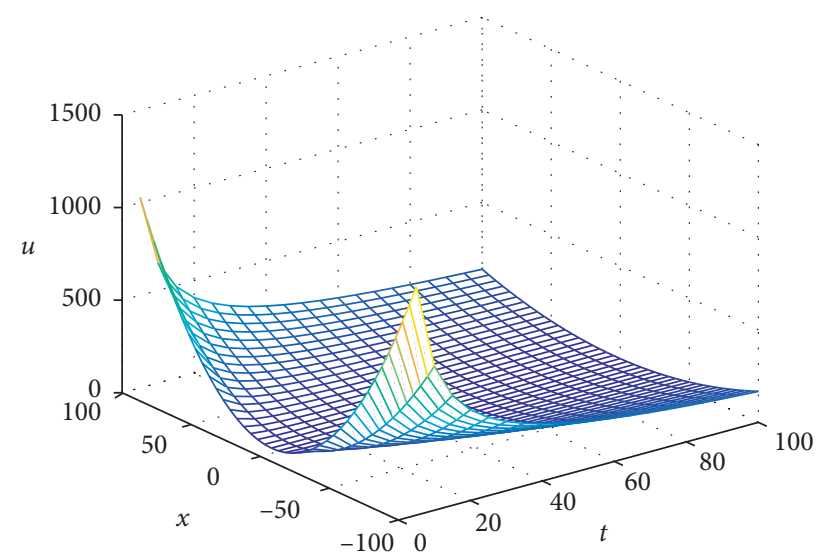

(b)

Figure 13: Dynamical profiles of solution (110) on the $(x, t, u)$-plane when (a) $\alpha=(1 / 3)$ and (b) $\alpha=(2 / 3)$.

$c_{0}=1, a_{1}=-2, b_{0}=1, c_{1}=2, c_{2}=1, c_{3}=-1.5, y=2, z=2$,

$t \in[0,10], x \in[-10,10]$, and $\quad x=2.5, y=2, z \in$ $[-10,10]$, the solutions (89) and (91) increase as $x$ and $y$ increases and decrease as time increases on both $(u, t, x)$ and $(u, t, y)$-planes; however, ones have exponential decay as $z \longrightarrow \infty$ when $x$ and $y$ are fixed. Under the Riemann-Liouville case:

As Figure 13 shows, taking $\alpha=(2 / 3), \mu_{1}= \pm(3 / 2), a_{0}=$ $2, p=r=1, b_{1}=-3, a_{1} f(z)+a_{1} g(y, z)+b_{0}=10, x \in$ $[-100,100], t \in[0,100]$, we see that

(i) If $\mu_{1}>0$, solutions (110) and (115) will blow up as time-space tends to infinity.

(ii) If $\mu_{1}<=0$, solutions (110) and (115) increase as $x$ increases if $y$ and $z$ are fixed and decay at $\max \left\{\mu_{1}, \alpha\right\}$ rate as $t \longrightarrow \infty$ if the spatial variables are fixed.

\section{Conclusions}

In this paper, by taking advantage of the fractional calculus methods, we avoid the invalid chain rule and suggest the new types of HBPs to solve some multidimensional time-fractional PDEs whose exact solutions were hardly obtained before. We get some explicit solutions of the $(2+1)$-dimensional KP equation, $(3+1)$-dimensional $\mathrm{ZK}$ equation, and JM equation by solving the reduced PDE system in both Riemann-Liouville and Caputo cases. These solutions are all in the general separated variable forms of new type which even include arbitrary functions, and the singularity solutions only appear in the Riemann-Liouville case. Furthermore, the dynamical analysis and long-time behaviors of these solutions are also performed.

However, to the best of our knowledge, that is far from enough, the HBP is only applicable to solve some special fractional nonlinear PDEs which satisfy the balance conditions (see Section 3.1) and get a few special exact solutions but not more general solutions. For even more multidimensional fractional nonlinear partial differential systems, there is still no better way to acquire their exact solutions generally at present. Nevertheless, finding more effective methods for constructing more exact solutions of more general $(N+1)$-dimensional fnPDEs will be quite a meaningful and challenging task in the future.

\section{Data Availability}

No data were used to support this study.

\section{Conflicts of Interest}

The authors declare that they have no conflicts of interest.

\section{Acknowledgments}

This work was supported by the NSFC under grant nos. 11775047 and 11871396.

\section{References}

[1] K. S. Miller and B. Ross, An Introductions to Fractional Calculus and Fractional Differential Equations, Wiley, New York, NY, USA, 1993.

[2] V. Kiryakova, "Generalized fractional calculus and applications," in Pitman Research Notes in Mathematics, Vol. 301, Wiley, New York, NY, USA, 1994.

[3] R. Hilfer, Applications of Fractional Calculus in Physics, World Scientific, Singapore, 2000.

[4] A. A. Kilbas, H. M. Srivastava, and J. J. Trujillo, "Theory and applications of fractional differential equations," in NorthHolland Mathematics Studies, Vol. 204, Elsevier, Amsterdam, Netherlands, 2006.

[5] I. Grigorenko and E. Grigorenko, "Chaotic dynamics of the fractional Lorenz system," Physical Review Letters, vol. 91, no. 3, Article ID 034101, 2003.

[6] D. Tripathi, S. K. Pandey, and S. Das, "Peristaltic flow of viscoelastic fluid with fractional Maxwell model through a channel," Applied Mathematics and Computation, vol. 215, no. 10, pp. 3645-3654, 2010.

[7] E. Sejdić, I. Djurović, and L. Stanković, "Fractional Fourier transform as a signal processing tool: an overview of recent developments," Signal Process, vol. 91, no. 6, pp. 1351-1369, 2011. 
[8] A. W. Lohmann, D. Mendlovic, Z. Zalevsky, and R. G. Dorsch, "Some important fractional transformations for signal processing," Optics Communications, vol. 125, no. 1-3, pp. 18-20, 1996.

[9] D. Baleanu, J. A. T. Machado, and A. C. Luo, Fractional Dynamics and Control, Springer Science and Business Media, Berlin, Germany, 2011.

[10] R. Sakthivel, N. I. Mahmudov, and J. J. Nieto, "Controllability for a class of fractional-order neutral evolution control systems," Applied Mathematics and Computation, vol. 218, no. 20, pp. 1033-1040, 2012.

[11] R. Metzler, E. Barkai, and J. Klafter, “Anomalous diffusion and relaxation close to thermal equilibrium: a fractional FokkerPlanck equation approach," Physical Review Letters, vol. 82, no. 18 , pp. 3563-3567, 1999.

[12] R. Metzler, W. G. Glöckle, and T. F. Nonnenmacher, "Fractional model equation for anomalous diffusion," Physica A: Statistical Mechanics and Its Applications, vol. 211, no. 1, pp. 13-24, 1994.

[13] B. I. Henry, T. A. M. Langlands, and S. L. Wearne, "Anomalous diffusion with linear reaction dynamics: from continous time random walks to fractional reaction-diffusion equations," Physical Review E, vol. 74, no. 3, Article ID 031116, 2006.

[14] V. V. Anh and N. N. Leonenko, "Scaling laws for fractional diffusion-wave equations with singular data," Statistics \& Probability Letters, vol. 48, no. 3, pp. 239-252, 2000.

[15] S. Zhai, Z. Weng, D. Gui, and X. Feng, "High-order compact operator splitting method for three-dimensional fractional equation with subdiffusion," International Journal of Heat and Mass Transfer, vol. 84, pp. 440-447, 2015.

[16] E. Ahmed and A. S. Elgazzar, "On fractional order differential equations model for nonlocal epidemics," Physica A: Statistical Mechanics and Its Applications, vol. 379, no. 2, pp. 607-614, 2007.

[17] F. Liu and K. Burrage, "Novel techniques in parameter estimation for fractional dynamical models arising from biological systems," Computers \& Mathematics with Applications, vol. 62, no. 3, pp. 822-833, 2011.

[18] M. EL-Shahed, "MHD of a fractional viscoelastic fluid in a circular tube," Mechanics Research Communications, vol. 33, no. 2, pp. 261-268, 2006.

[19] A. A. Zafar, D. Vieru, and S. Akhtar, "Magnetohydrodynamics of rotating fractional second grade fluid in porous medium," Journal of Prime Research in Mathematics, vol. 10, pp. 45-58, 2015.

[20] I. Podlubny, "Fractional differential equations," in Mathematics in Science and Engineering, Academic Press, Cambridge, MA, USA, 1999.

[21] S. Wojciech and Z. V. George, "A hyperelastic fractional damage material model with memory," International Journal of Solids and Structures, vol. 124, pp. 151-160, 2017.

[22] C. S. Drapaca and S. Sivaloganathan, "A fractional model of continuum mechanics," Journal of Elasticity, vol. 107, pp. 107-123, 2012.

[23] W. Sumelka, "Non-local Kirchhoff-Love plates in terms of fractional calculus," Archives of Civil and Mechanical Engineering, vol. 15, no. 1, pp. 231-242, 2015.

[24] K. A. Lazopoulos and A. K. Lazopoulos, "Fractional vector calculus and fluid mechanics," Journal of the Mechanical Behavior of Materials, vol. 26, no. 1-2, pp. 43-54, 2017.

[25] Z. Rahimi, G. Rezazadeh, and W. Sumelka, "A study of critical point instability of micro and nano beams under a distributed variable-pressure force in the framework of the inhomogeneous non-linear nonlocal theory," Archives of Mechanics, vol. 69, no. 6, pp. 413-433, 2017.

[26] B. B. Peter, "Dynamical systems approach of internal length in fractional calculus," Engineering Transactions, vol. 65, no. 1, pp. 209-215, 2017.

[27] V. Daftardar-Gejji and H. Jafari, "Adomian decomposition: a tool for solving a system of fractional differential equations," Journal of Mathematical Analysis and Applications, vol. 301, no. 2, pp. 508-518, 2005.

[28] T. Bakkyaraj and R. Sahadevan, "An approximate solution to some classes of fractional nonlinear partial differential equation using adomain decomposition method," Journal of Fractional Calculus and Applications, vol. 5, no. 1, pp. 37-52, 2014.

[29] T. Bakkyaraj and R. Sahadevan, "Approximate analytical solution of two coupled time fractional nonlinear schrödinger equations," International Journal of Applied and Computational Mathematics, vol. 2, no. 1, pp. 113-135, 2016.

[30] T. Bakkyaraj and R. Sahadevan, "On solutions of two coupled fractional time derivative Hirota equations," Nonlinear Dynamics, vol. 77, no. 4, pp. 1309-1322, 2014.

[31] Z. Odibat and S. Momani, "The variational iteration method: an efficient scheme for handling fractional partial differential equations in fluid mechanics," Computers \& Mathematics with Applications, vol. 58, no. 11-12, pp. 2199-2208, 2009.

[32] G.-C. Wu and E. W. M. Lee, "Fractional variational iteration method and its application," Physics Letters A, vol. 374, no. 25, pp. 2506-2509, 2010.

[33] R. K. Gazizov, A. A. Kasatkin, and S. Y. Lukashchuk, "Continuous transformation groups of fractional differential equation," Vestnik USATU, vol. 93, no. 21, pp. 125-135, 2007.

[34] R. K. Gazizov and A. A. Kasatkin, "Symmetry properties of fractional diffusion equations," Physica Scripta, vol. T136, Article ID 014016, 2009.

[35] R. K. Gazizov, A. A. Kasatkin, and S. Y. Lukashchuk, "Group invariant solutions of fractional differential equations," in Nonlinear Science and Complexity, J. Machado, A. Luo, R. Barbosa et al., Eds., pp. 51-58, Springer, Berlin, Germany, 2011.

[36] A. A. Kasatkin, "Symmetry properties for systems of two ordinary fractional differential equations," Ufa Mathematical Journal, vol. 4, pp. 65-75, 2012.

[37] T. Bakkyaraj and R. Sahadevan, "Invariant analysis of nonlinear fractional ordinary differential equations with Riemann-Liouville fractional derivative," Nonlinear Dynamics, vol. 80, no. 1-2, pp. 447-455, 2015.

[38] R. Sahadevan and T. Bakkyaraj, "Invariant analysis of time fractional generalized Burgers and Korteweg-de vries equations," Journal of Mathematical Analysis and Applications, vol. 393, no. 2, pp. 341-347, 2012.

[39] Q. Huang and R. Zhdanov, "Symmetries and exact solutions of the time fractional Harry-Dym equation with RiemannLiouville derivative," Physica A: Statistical Mechanics and Its Applications, vol. 409, pp. 110-118, 2014.

[40] R. Sahadevan and P. Prakash, "On Lie symmetry analysis and invariant subspace methods of coupled time fractional partial differential equations," Chaos, Solitons \& Fractals, vol. 104, pp. 107-120, 2017.

[41] R. K. Gazizov and A. A. Kasatkin, "Construction of exact solutions for fractional order differential equations by the invariant subspace method," Computers \& Mathematics with Applications, vol. 66, no. 5, pp. 576-584, 2013.

[42] R. Sahadevan and T. Bakkyaraj, "Invariant subspace method and exact solutions of certain nonlinear time fractional partial 
differential equations," Fractional Calculus and Applied Analysis, vol. 18, pp. 146-162, 2015.

[43] R. Sahadevan and P. Prakash, "Exact solution of certain time fractional nonlinear partial differential equations," Nonlinear Dynamics, vol. 85, no. 1, pp. 659-673, 2016.

[44] R. Sahadevan and P. Prakash, "Exact solutions and maximal dimension of invariant subspaces of time fractional coupled nonlinear partial differential equations," Communications in Nonlinear Science and Numerical Simulation, vol. 42, pp. 158-177, 2017.

[45] W. Rui, "Applications of homogenous balanced principle on investigating exact solutions to a series of time fractional nonlinear PDEs," Communications in Nonlinear Science and Numerical Simulation, vol. 47, pp. 253-266, 2017.

[46] W. Rui, "Applications of integral bifurcation method together with homogeneous balanced principle on investigating exact solutions of time fractional nonlinear PDEs," Nonlinear Dynamics, vol. 91, no. 1, pp. 697-712, 2018.

[47] C. Wu and W. Rui, "Method of separation variables combined with homogenous balanced principle for searching exact solutions of nonlinear time-fractional biological population model," Communications in Nonlinear Science and Numerical Simulation, vol. 63, pp. 88-100, 2018.

[48] Y. R. Molliq, M. S. M. Noorani, I. Hashim, and R. R. Ahmad, "Approximate solutions of fractional Zakharov-Kuznetsov equations by VIM," Journal of Computational and Applied Mathematics, vol. 233, no. 2, pp. 103-108, 2009.

[49] H. Jafari and S. Seifi, "Solving a system of nonlinear fractional partial differential equations using homotopy analysis method," Communications in Nonlinear Science and Numerical Simulation, vol. 14, no. 5, pp. 1962-1969, 2009.

[50] S. S. Ray and S. Sahoo, "Invariant analysis and conservation laws of $(2+1)$ dimensional time-fractional ZK-BBM equation in gravity water waves," Computers \& Mathematics with Applications, vol. 75, pp. 2271-2279, 2018.

[51] J.-H. He, S. K. Elagan, and Z. B. Li, "Geometrical explanation of the fractional complex transform and derivative chain rule for fractional calculus," Physics Letters A, vol. 376, no. 4, pp. 257-259, 2012.

[52] V. E. Tarasov, "On chain rule for fractional derivatives," Communications in Nonlinear Science and Numerical Simulation, vol. 30, no. 1-3, pp. 1-4, 2016.

[53] X. Wang and Y. Chen, "Darboux transformations and N -soliton solutions of two $(2+1)$-dimensional nonlinear equations," Communications in Theoretical Physics, vol. 61, no. 4, pp. 423-430, 2014.

[54] W.-X. Ma, "Lump-type solutions to the $(3+1)$-dimensional jimbo-miwa equation," International Journal of Nonlinear Sciences and Numerical Simulation, vol. 17, no. 7-8, 2016.

[55] M. Wang and X. Li, "Extended F-expansion method and periodic wave solutions for the generalized Zakharov equations," Physics Letters A, vol. 343, no. 1-3, pp. 48-54, 2005.

[56] C. Zhu and C. Qu, "Maximal dimension of invariant subspaces admitted by nonlinear vector differential operators," Journal of Mathematical Physics, vol. 52, Article ID 043507, 2011. 